\title{
Integrability of the constrained rigid body
}

\author{
Jaume Llibre, Rafael Ramírez and \\ Natalia Sadovskaia
}

\begin{abstract}
The integrability theory for the differential equations which describe the motion of an unconstrained rigid body around a fixed point is well known. When there are constraints the theory of integrability is incomplete. The main objective of this paper is to analyze the integrability of the equations of motion of a constrained rigid body around a fixed point in a force field with potential $U(\gamma)=U\left(\gamma_{1}, \gamma_{2}, \gamma_{3}\right)$. This motion subject to the constraint $\langle\nu, \omega\rangle=0$ with $\nu$ is a constant vector is known as the Suslov problem, and when $\nu=\gamma$ is the known Veselova problem, here $\omega=\left(\omega_{1}, \omega_{2}, \omega_{3}\right)$ is the angular velocity and $\langle$,$\rangle is the inner product of \mathbb{R}^{3}$.
\end{abstract}

We provide the following new integrable cases.

(i) The Suslov's problem is integrable under the assumption that $\nu$ is an eigenvector of the inertial tensor $I$ and the potential is such that

$$
U=-\frac{1}{2 I_{1} I_{2}}\left(I_{1} \mu_{1}^{2}+I_{2} \mu_{2}^{2}\right),
$$

where $I_{1}, I_{2}$ and $I_{3}$ are the principal moments of inertia of the body, $\mu_{1}$ and $\mu_{2}$ are solutions of the first order partial differential equation

$$
\gamma_{3}\left(\frac{\partial \mu_{1}}{\partial \gamma_{2}}-\frac{\partial \mu_{2}}{\partial \gamma_{1}}\right)-\gamma_{2} \frac{\partial \mu_{1}}{\partial \gamma_{3}}+\gamma_{1} \frac{\partial \mu_{2}}{\partial \gamma_{3}}=0
$$

Jaume Llibre

Departament de Matemàtiques, Universitat Autònoma de Barcelona, 08193 Bellaterra, Barcelona, Catalonia, Spain.

E-mail: jllibre@mat.uab.cat

Rafael Ramírez

Departament d'Enginyeria Informàtica i Matemàtiques, Universitat Rovira i Virgili, Avinguda dels Països Catalans 26, 43007 Tarragona, Catalonia, Spain.

E-mail: rafaelorlando.ramirez@urv.cat

Natalia Sadovskaia

Departament de Matemàtica Aplicada II, Universitat Politècnica de Catalunya, C. Pau Gargallo 5, 08028 Barcelona, Catalonia, Spain.

E-mail: natalia.sadovskaia@upc.edu 
(ii) The Veselova problem is integrable for the potential

$$
U=-\frac{\Psi_{1}^{2}+\Psi_{2}^{2}}{2\left(I_{1} \gamma_{2}^{2}+I_{2} \gamma_{1}^{2}\right)}
$$

where $\Psi_{1}$ and $\Psi_{2}$ are the solutions of the first order partial differential equation

$\left(I_{2}-I_{1}\right) \gamma_{1} \gamma_{2}\left\langle\gamma, \frac{\partial \Psi_{2}}{\partial \gamma}\right\rangle+I_{1} \gamma_{2} \frac{\partial \Psi_{2}}{\partial \gamma_{1}}-I_{2} \gamma_{1} \frac{\partial \Psi_{2}}{\partial \gamma_{2}}-p\left(\gamma_{3}\left\langle\gamma, \frac{\partial \Psi_{1}}{\partial \gamma}\right\rangle-\frac{\partial \Psi_{1}}{\partial \gamma_{3}}\right)=0$

where $p=\sqrt{I_{1} I_{2} I_{3}\left(\frac{\gamma_{1}^{2}}{I_{1}}+\frac{\gamma_{2}^{2}}{I_{2}}+\frac{\gamma_{3}^{2}}{I_{3}}\right)}$.

Also it is integrable when the potential $U$ is a solution of the second order partial differential equation

$$
2 \frac{\partial U}{\partial \tau_{3}}+I_{1} I_{2} I_{3} \frac{\partial^{2} U}{\partial \tau_{2}^{2}}+\left(\tau_{2}-I_{1}-I_{2}-I_{3}\right) \frac{\partial^{2} U}{\partial \tau_{3} \partial \tau_{2}}+\tau_{3} \frac{\partial^{2} U}{\partial \tau_{3}^{2}}=0
$$

where $\tau_{2}=I_{1} \gamma_{1}^{2}+I_{2} \gamma_{2}^{2}+I_{3} \gamma_{3}^{2}$ and $\tau_{3}=\frac{\gamma_{1}^{2}}{I_{1}}+\frac{\gamma_{2}^{2}}{I_{2}}+\frac{\gamma_{3}^{2}}{I_{3}}$.

Moreover we show that these integrable cases contain as a particular case the previous known results.

Keywords ordinary differential equation · invariant measure $\cdot$ mechanical systems - nonholonomic system · constraint · rigid body $\cdot$ Suslov problem . Veselova problem · integrability

Mathematics Subject Classification (2000) 14P25 - 34C05 - 34A34

\section{Introduction}

For simplicity we shall assume that all the functions which appear in this paper are of class $\mathcal{C}^{\infty}$, although most of the results remain valid under weaker smoothness.

Consider the differential system

$$
\dot{\mathbf{x}}=\mathcal{X}(\mathbf{x}), \quad x=\left(x_{1}, \ldots, x_{N}\right) \in \mathbb{R}^{N} .
$$

Let $\Omega$ be an open and dense subset of $\mathbb{R}^{N}$. A non-constant function $\Phi: \Omega \longrightarrow$ $\mathbb{R}$ such that $\Phi$ is constant on the solutions of system (1) contained in $\Omega$ is called a first integral. We say that system (1) is explicitly integrable in $\Omega$ if it has $\Phi_{k}: \Omega \longrightarrow \mathbb{R}$ for $k=1, \ldots, N-1$ functionally independent first integrals, i.e. the rank of the $(N-1) \times N$ Jacobian matrix

$$
\frac{\partial\left(\Phi_{1}, \ldots, \Phi_{N-1}\right)}{\partial\left(x_{1}, \ldots, x_{N}\right)}
$$


is $N-1$ in all the points $\left(x_{1}, \ldots, x_{N}\right)$ of $\Omega$ except perhaps in a zero Lebesgue measure set.

Let $\Sigma$ be an open subset of $\mathbb{R}^{M}$ and let $F_{j}: \Omega \times \Sigma \longrightarrow \mathbb{R}$ for $j=1, \ldots, M$ be a smooth maps. The relation $F_{j}:=F_{j}\left(\mathbf{x}, K_{1}, \ldots, K_{M}\right)=0$, with $K_{1}, \ldots, K_{M}$ constants, is called a general integral of (1) if $\left.\mathcal{X} F_{j}\right|_{F_{1}=\ldots=F_{M}=0}=0$. System (1) is implicitly integrable if it admits $M=N-1$ general integrals $F_{j}=0$, for $j=1, \ldots, N-1$ such that the rank of $(N-1) \times(N-1)$ Jacobian matrix

$$
\frac{\partial\left(F_{1}, \ldots, F_{N-1}\right)}{\partial\left(K_{1}, \ldots, K_{N-1}\right)}
$$

is $N-1$ in all the points $\left(\mathbf{x}, K_{1}, \ldots, K_{N-1}\right)$ of $\Omega \times \Sigma$ except perhaps in a zero Lebesgue measure set. Indeed, under condition (2) and by the Implicit Function Theorem, we can obtain from the set of $N-1$ general integrals $N-1$ local first integrals of the form $K_{j}=\Phi_{j}(\mathbf{x})$. Consequently system (1) is locally explicitly integrable in view of the previous definition.

The integration theory of the differential equations which describe the motion of mechanical systems with nonintegrable constraints (i.e. nonholonomic system) is not so complete as for the unconstrained systems (i.e. holonomic systems). This is due to several reasons. One of them is that the equations of motion of nonholonomic systems in general have no invariant measure, as they have the equations of motion of holonomic systems, see for instance [12].

The existence of an invariant measure simplifies the integration of the differential equations. It is well known the Euler-Jacobi Theorem : If the differential system (1) has $N-2$ independent first integrals $\Phi_{1}, \ldots, \Phi_{N-2}$ and

$$
\operatorname{div}(M(\mathbf{x}) \mathcal{X}(\mathbf{x}))=\sum_{j=1}^{N} \frac{\partial\left(M(\mathbf{x}) \mathcal{X}_{j}\right)}{\partial x_{j}}=0
$$

for a convenient function $M(\mathbf{x})>0$, then the differential system is explicitly integrable. We observe that this condition using the divergence is necessary and sufficient for the existence of an invariant measure with respect to the action of the vector field (1) (due to Euler-Jacobi Theorem, see for instance $[10,12])$.

Let $\bar{\Sigma}$ be an open subset of $\mathbb{R}^{N-2}$. System (1) is quasi-implicitly integrable in $\Omega \times \bar{\Sigma}$ if it has an invariant measure and admits $N-2$ general integrals $F_{j}\left(\mathbf{x}, K_{1}, \ldots, K_{N-2}\right)=0$ for $j=1, \ldots, N-2$ such that the rank of $(N-2) \times$ $(N-2)$ Jacobian matrix

$$
\frac{\partial\left(F_{1}, \ldots, F_{N-2}\right)}{\partial\left(K_{1}, \ldots, K_{N-2}\right)}
$$

is $N-2$, in all the points $\left(x_{1}, \ldots, x_{N}\right)$ of $\Omega$ except perhaps in a zero Lebesgue measure set and for arbitrary constants $K_{1}, \ldots, K_{N-2}$.

Now we study the integrability theory for the motion of a rigid body around a fixed point. If this mechanical system is free of constraints then its integrability it is well known (see for instance [1]). But the integration of the equations 
of motion of this mechanical system with constraints is incomplete. For example the integrability is in general unknown when the constraint is of the form

$$
\langle\nu, \omega\rangle=0,
$$

where $\nu=\nu(\gamma)=\left(\nu_{1}, \nu_{2}, \nu_{3}\right)$ is a vector of $\mathbb{R}^{3}, \gamma$ is the unit vector of a spatially fixed axis in the coordinate system rigidly connected with the body and such that

$$
\gamma=\left(\gamma_{1}, \gamma_{2}, \gamma_{3}\right)=(\sin z \sin x, \sin z \cos x, \cos z),
$$

$(x, y, z)=(\varphi, \psi, \theta)$ are the Euler angles and $\omega=\left(\omega_{1}, \omega_{2}, \omega_{3}\right)$ is the angular velocity.

Applying the method of Lagrange multipliers the equations of motion of the rigid body around a fixed point with the constraint (3) are (for more details see for instance $[1,4]$ )

$$
I \dot{\omega}=I \omega \wedge \omega+\gamma \wedge \frac{\partial U}{\partial \gamma}+\mu \nu, \quad \dot{\gamma}=\gamma \wedge \omega, \quad\langle\nu, \omega\rangle=0,
$$

where $I$ is the inertial tensor of the rigid body, i.e.

$$
I=\left(\begin{array}{lll}
I_{11} & I_{12} & I_{13} \\
I_{12} & I_{22} & I_{23} \\
I_{13} & I_{23} & I_{33}
\end{array}\right),
$$

$U=U\left(\gamma_{1}, \gamma_{2}, \gamma_{3}\right)$ is the potential function, $\frac{\partial U(\gamma)}{\partial \gamma}$ is the gradient of $U(\gamma)$ with respect to $\gamma$, and $\wedge$ is the "wedge" product in $\mathbb{R}^{3}$. .

We observe that the equations $\dot{\gamma}=\gamma \wedge \omega$ are known as the Poisson differential equations.

System (5) always has three independent first integrals, namely

$$
\Phi_{1}=\langle\gamma, \gamma\rangle=\gamma_{1}^{2}+\gamma_{2}^{2}+\gamma_{3}^{2}, \quad \Phi_{2}=\langle\nu, \omega\rangle, \quad \Phi_{3}=\frac{1}{2}\langle I \omega, \omega\rangle+U(\gamma)
$$

We shall study two particular cases with the constraint (3): the Suslov problem $(\nu=\mathbf{a})$ and the Veselova problem $(\nu=\gamma)$. The main objective of this paper is to present new cases of integrability for these two problems which contains as particular cases the previous results on the integrability of these two problems.

\subsection{The Suslov problem}

The Suslov problem is the study of the motion of a rigid body around a fixed point and subject to the nonholonomic constraint $\langle\mathbf{a}, \omega\rangle=0$, where $\mathbf{a}$ is a constant vector (see [19]). Suppose that the body rotates in a force field with 
potential $U(\gamma)=U\left(\gamma_{1}, \gamma_{2}, \gamma_{3}\right)$. Applying the method of Lagrange multipliers the equations of motion are

$$
I \dot{\omega}=I \omega \wedge \omega+\gamma \wedge \frac{\partial U}{\partial \gamma}+\mu \mathbf{a}, \quad \dot{\gamma}=\gamma \wedge \omega, \quad\langle\mathbf{a}, \omega\rangle=0,
$$

System (7) always has the three independent first integrals (6) with $\nu=\mathbf{a}$.

In order to have real motions we must take $\Phi_{1}=1, \Phi_{2}=0$ in (6). In this case using the first integrals $\Phi_{3}$ we can reduce the problem of integration of (7) to the problem of existence of an invariant measure and a fourth independent first integral $\Phi_{4}$. Under these assumptions, by the Euler-Jacobi Theorem (see for instance $[10,5])$ the Suslov problem is integrable [13]. In general system (7) has no invariant measure if the vector $\mathbf{a}$ is not an eigenvector of the tensor of inertia. It is well-known the following result, see [12].

Proposition 1 If $\boldsymbol{a}$ is an eigenvector of the inertial tensor I, i.e.

$$
I \boldsymbol{a}=\kappa \boldsymbol{a}
$$

for some $\kappa \in \mathbb{R}$, then the flow of system (7) preserves the Lebesgue measure in $\mathbb{R}^{6}=\mathbb{R}^{3}\{\omega\} \times \mathbb{R}^{3}\{\gamma\}$.

Suslov in [19] has considered the case when the body is in absence of external forces, i.e. $U=0$. In this case the system

$$
I \dot{\omega}=I \omega \wedge \omega+\mu \mathbf{a}
$$

or equivalently (see [7])

$$
I \dot{\omega}=-\frac{\langle I \tilde{\mathbf{a}}, \omega\rangle}{\left\langle I^{-1} \tilde{\mathbf{a}}, \tilde{\mathbf{a}}\right\rangle}\left(I^{-1} \tilde{\mathbf{a}} \wedge \omega\right),
$$

can be solved with respect to $\omega$, i.e. it is integrable by quadratures. The analysis of theses quadratures shows that if (8) does not holds then all trajectories $\omega=\omega(t)$ approach asymptotically as $t \rightarrow \pm \infty$ to some fixed straight line on the plane $\langle\mathbf{a}, \omega\rangle=0$. Consequently equations (7) have no invariant measure . The question about the possibility to find $\gamma=\gamma(t)$ by quadratures in general remains open (for more details see [7]).

If (8) holds then along the solutions of (7) the kinetic moment $\langle I \omega, I \omega\rangle$ is a first integral (see [12]).

From now on we suppose that equality (8) is fulfilled.

Without loss of generality we can choose the vector a as the third axis, i.e. $\mathbf{a}=(0,0,1)$ and consequently the constrained becomes $\omega_{3}=\dot{x}+\dot{y} \cos z=0$. Then, the equations of motion are

$$
\begin{aligned}
& I_{1} \dot{\omega}_{1}=\gamma_{2} \frac{\partial U}{\partial \gamma_{3}}-\gamma_{3} \frac{\partial U}{\partial \gamma_{2}}, \quad I_{2} \dot{\omega}_{2}=\gamma_{3} \frac{\partial U}{\partial \gamma_{1}}-\gamma_{1} \frac{\partial U}{\partial \gamma_{2}} \\
& \dot{\gamma}_{1}=-\gamma_{3} \omega_{2}, \quad \dot{\gamma}_{2}=\gamma_{3} \omega_{1}, \quad \dot{\gamma}_{3}=\gamma_{1} \omega_{2}-\gamma_{2} \omega_{1},
\end{aligned}
$$


where $I=\operatorname{diag}\left(I_{1}, I_{2}, I_{3}\right)$, and $I_{k}$ are the principal moments of inertia of the body.

Kharlamova-Zabalina in [11] studied the case when the body rotates in the homogenous force field with the potential $U=\langle\mathbf{b}, \gamma\rangle$ where the vector $\mathbf{b}$ is orthogonal to the vector $\mathbf{a}$. Under these conditions the equations of motion have the first integral $\Phi_{4}=\langle I \omega, \mathbf{b}\rangle$.

Kozlov consider the case when $\mathbf{b}=\lambda \mathbf{a}, \lambda \neq 0$. The integrability problem in this case was study in $[12,16]$. If $I_{1} \neq I_{2}$, apparently the equations have no additional first integral independent of the energy integral. When $I_{1}=I_{2}$ and $U=\lambda\left\langle\mathbf{a}, \gamma_{3}\right\rangle$ there exists the fourth integral $\Phi_{4}=\omega_{1} \gamma_{1}+\omega_{2} \gamma_{2}$.

For the case $U=\lambda|I|\left\langle I^{-1} \gamma, \gamma\right\rangle$, where $|I|=\operatorname{det} I$, system (7) has the Klebsh-Tisserand first integral $\Phi_{4}=\frac{1}{2}\langle I \omega, I \omega\rangle-\frac{1}{2} \lambda|I|\left\langle I^{-1} \gamma, \gamma\right\rangle$ (see for instance [12]).

Okuneva in [17] proved the integrability of the Suslov problem for the potential $U=\alpha \gamma_{1}+\beta \gamma_{2}+\frac{\lambda}{2}\left\langle I^{-1} \gamma, \gamma\right\rangle$, where $\alpha, \beta$ and $\lambda$ are constants. The first integral is $\Phi_{4}=I_{1} \omega_{1}^{2}-\lambda\left(I_{2}-I_{3}\right) \gamma_{2}^{2}-2 \beta \gamma_{2}$, or equivalently $\Phi_{4}=$ $I_{2} \omega_{2}^{2}-\lambda\left(I_{3}-I_{1}\right) \gamma_{1}^{2}-2 \alpha \gamma_{1}$.

Dragovic et al. in [6] consider the case when the potential $U=c\left(\gamma_{1}, \gamma_{2}^{2}+\right.$ $\left.\gamma_{3}^{2}\right)-d\left(\gamma_{2}, \gamma_{1}^{2}+\gamma_{3}^{2}\right)$ for arbitrary functions $c=c\left(\gamma_{1}, \gamma_{2}^{2}+\gamma_{3}^{2}\right)$ and $d=d\left(\gamma_{2}, \gamma_{1}^{2}+\right.$ $\left.\gamma_{3}^{2}\right)$, proved that $\Phi_{4}=\frac{1}{2}\langle I \omega, I \omega\rangle+I_{2} c\left(\gamma_{1}, \gamma_{2}^{2}+\gamma_{3}^{2}\right)-I_{1} d\left(\gamma_{2}, \gamma_{1}^{2}+\gamma_{3}^{2}\right)$ is a first integral of system (7).

\subsection{The Veselova problem}

The Veselova problem describes the motion of a rigid body which rotates around a fixed point and subject to the nonholonomic constraint

$$
\langle\gamma, \omega\rangle=\dot{y}+\dot{x} \cos z=0,
$$

Thus, in the case of the Veselova constraint the projection of the angular velocity to a spatially fixed axis is zero.

Suppose that the body rotates in a force field with potential $U\left(\gamma_{1}, \gamma_{2}, \gamma_{3}\right)$. Applying the method of Lagrange multipliers we write the equations of motion in the form

$$
I \dot{\omega}=I \omega \wedge \omega+\gamma \wedge \frac{\partial U}{\partial \gamma}+\mu \gamma, \quad \dot{\gamma}=\gamma \wedge \omega, \quad\langle\gamma, \omega\rangle=0,
$$

where $I=\operatorname{diag}\left(I_{1}, I_{2}, I_{3}\right)$. System (11) has always three independent integrals (6) with $\nu=\gamma$.

As proved in [20] system (11) has an invariant measure with density

$$
\sqrt{\frac{\gamma_{1}^{2}}{I_{1}}+\frac{\gamma_{2}^{2}}{I_{2}}+\frac{\gamma_{3}^{2}}{I_{3}}}
$$


Thus, in view of the Euler-Jacobi Theorem we obtain that if there exists a fourth first integrals $\Phi_{4}$ independent with $\Phi_{1}, \Phi_{2}, \Phi_{3}$, then the Veselova problem is integrable. In order to have real motions we must take $\Phi_{1}=1$ and $\Phi_{2}=0$.

Remark 1 From (11) we get the equalities

$$
\begin{aligned}
\frac{d}{d t}(\gamma \wedge I \omega) & =\frac{d \gamma}{d t} \wedge I \omega+\gamma \wedge I \frac{d \omega}{d t} \\
& =(\gamma \wedge \omega) \wedge I \omega+\gamma \wedge\left(I \omega \wedge \omega+\gamma \wedge \frac{\partial U}{\partial \gamma}+\lambda \gamma\right) \\
& =(\gamma \wedge \omega) \wedge I \omega+(\omega \wedge I \omega) \wedge \gamma+\gamma \wedge\left(\gamma \wedge \frac{\partial U}{\partial \gamma}\right),
\end{aligned}
$$

and by considering the identities

$$
a \wedge(b \wedge c)=\langle a, c\rangle b-\langle a, b\rangle c, \quad a \wedge(b \wedge c)+b \wedge(c \wedge a)=-c \wedge(a \wedge b),
$$

we obtain

$$
\frac{d}{d t}(\gamma \wedge I \omega)=-\frac{\partial U}{\partial \gamma}+\gamma\left(\left\langle\gamma, \frac{\partial U}{\partial \gamma}\right\rangle-\langle I \omega, \omega\rangle\right)
$$

From this relation we deduce the equation

$$
\frac{d(p \omega)}{d t}=\frac{1}{p}\left(\gamma \wedge I \frac{\partial U}{\partial \gamma}+I \gamma \wedge \gamma\left(\left\langle\gamma, \frac{\partial U}{\partial \gamma}\right\rangle-\langle I \omega, \omega\rangle\right)\right)
$$

where $p=\sqrt{I_{1} I_{2} I_{3}\left(\frac{\gamma_{1}^{2}}{I_{1}}+\frac{\gamma_{2}^{2}}{I_{2}}+\frac{\gamma_{3}^{2}}{I_{3}}\right)}$.

\section{Statement of the main results}

Our first main result is the following.

Theorem 1 The motion of the rigid body subject to the nonholonomic constraint $\omega_{3}=0$ (Suslov problem), under assumption (8) is described by system (9). We suppose that the rigid body rotates under the action of a force field defined by the potential

$$
U=-\frac{1}{2 I_{1} I_{2}}\left(I_{1} \mu_{1}^{2}+I_{2} \mu_{2}^{2}\right)
$$

where $\mu_{1}=\mu_{1}\left(\gamma_{1}, \gamma_{2}, \gamma_{3}, K_{3}, K_{4}\right)$ and $\mu_{2}=\mu_{2}\left(\gamma_{1}, \gamma_{2}, \gamma_{3}, K_{3}, K_{4}\right)$ with $K_{3}$ and $K_{4}$ constants. Assume that $\mu_{1}$ and $\mu_{2}$ are solutions of the first order partial differential equation

$$
\gamma_{3}\left(\frac{\partial \mu_{1}}{\partial \gamma_{2}}-\frac{\partial \mu_{2}}{\partial \gamma_{1}}\right)-\gamma_{2} \frac{\partial \mu_{1}}{\partial \gamma_{3}}+\gamma_{1} \frac{\partial \mu_{2}}{\partial \gamma_{3}}=0
$$


satisfying

$$
\frac{\partial \mu_{1}}{\partial K_{3}} \frac{\partial \mu_{2}}{\partial K_{4}}-\frac{\partial \mu_{2}}{\partial K_{3}} \frac{\partial \mu_{1}}{\partial K_{4}} \neq 0 \quad \text { for all }\left(\gamma_{1}, \gamma_{2}, \gamma_{3}\right) \in \mathbb{R}^{3}
$$

Then the following statements hold.

(a) System (9) has the general integrals

$$
\begin{aligned}
& F_{1}=I_{1} \omega_{1}-\mu_{2}\left(\gamma_{1}, \gamma_{2}, \gamma_{3}, K_{3}, K_{4}\right)=0 \\
& F_{2}=I_{2} \omega_{2}+\mu_{1}\left(\gamma_{1}, \gamma_{2}, \gamma_{3}, K_{3}, K_{4}\right)=0
\end{aligned}
$$

Moreover system (9) is quasi-implicitly integrable.

(b) The first integrals of Suslov, Kharlamova-Zabelina's, Kozlov, Dragović-GajićJovanović, Klebsh-Tisserand and Tisserand-Okuneva are particular cases of the first integrals of statement $(a)$.

(c) Using (17) the Poisson equations for the Suslov problem take the form

$$
\dot{\gamma}_{1}=-\gamma_{3} \frac{\mu_{1}}{I_{2}}, \quad \dot{\gamma}_{2}=-\gamma_{3} \frac{\mu_{2}}{I_{1}}, \quad \dot{\gamma}_{3}=-\gamma_{1} \frac{\mu_{1}}{I_{2}}-\gamma_{2} \frac{\mu_{2}}{I_{1}} .
$$

We provide the solution $\gamma(t)=\left(\gamma_{1}(t), \gamma_{2}(t), \gamma_{3}(t)\right)$ for all the cases of statement $(b)$.

The proof of Theorem 1 is given in section 2 .

We note that the potential of the equation (14) contains as particular cases the potentials studied by the authors Suslov, Kharlamova-Zabelina's, Kozlov, Dragović-Gajić-Jovanović, Klebsh-Tisserand and Tisserand-Okuneva, previously defined.

Remark 2 It is easy to check that the functions

$$
\begin{aligned}
& \mu_{1}=\frac{\partial \tilde{S}\left(\gamma_{1}, \gamma_{2}, \gamma_{3}, K_{3}, K_{4}\right)}{\partial \gamma_{1}}+\Psi_{1}\left(\gamma_{2}^{2}+\gamma_{3}^{2}, \gamma_{1}, K_{3}, K_{4}\right), \\
& \mu_{2}=\frac{\partial \tilde{S}\left(\gamma_{1}, \gamma_{2}, \gamma_{3}, K_{3}, K_{4}\right)}{\partial \gamma_{2}}+\Psi_{2}\left(\gamma_{1}^{2}+\gamma_{3}^{2}, \gamma_{2}, K_{3}, K_{4}\right),
\end{aligned}
$$

are solutions of (15), where

$\tilde{S}\left(\gamma_{1}, \gamma_{2}, \gamma_{3}, K_{3}, K_{4}\right)=S\left(\gamma_{1}, \gamma_{2}, K_{3}, K_{4}\right)+\int \Upsilon\left(\gamma_{1}^{2}+\gamma_{2}^{2}, \gamma_{3}, K_{3}, K_{4}\right) d\left(\gamma_{1}^{2}+\gamma_{2}^{2}\right)$,

and $S, \Psi_{1}, \Psi_{2}$ and $\Upsilon$ are arbitrary smooth functions for which (16) holds.

Remark 2 will be used for proving statement (b) of Theorem 1.

Note that in Theorem 1 we are working with two general integrals because we are only using the two first integrals $\Phi_{1}=\langle\gamma, \gamma\rangle=1$ and $\Phi_{2}=\langle\omega, \gamma\rangle=0$. There are two main reasons for working with two general integrals instead of using also the energy integral $\Phi_{3}=\langle I \omega, \omega\rangle+U(\gamma)$, which would allow to look for a unique general integral for determining the integrability of the Suslov 
problem. The first reason is that working with two general integrals all the computations for studying the integrable cases are easier. The second reason is that working with two general integrals, which are linear with respect to the angular velocity, the Poisson differential equations can be written in the form (18) which do not depend on the angular velocity, and consequently its integrability is in general easier, mainly in the cases of statement (b) (for more details see [18]).

Remark 3 The solutions of (15) can be represented in a formal Laurent series

$$
\mu_{1}=\sum_{n, j, k \in \mathbb{Z}} a_{n j k} \gamma_{1}^{n} \gamma_{2}^{j} \gamma_{3}^{k}, \quad \mu_{2}=\sum_{n, j, k \in \mathbb{Z}} b_{n j k} \gamma_{1}^{n} \gamma_{2}^{j} \gamma_{3}^{k},
$$

where $a_{n j k}=a_{n j k}\left(K_{3}, K_{4}\right)$ and $b_{n j k}=b_{n j k}\left(K_{3}, K_{4}\right)$ are coefficients which satisfy the equalities

$$
j a_{n j k}-(n+1) b_{n+1, j-1, k}=(k+2)\left(a_{n, j-2, k+2}-b_{n-1, j-1, k+2}\right),
$$

where $n, j, k \in \mathbb{Z}$.

Our second result on the Veselova problem is the following.

Theorem 2 The motion of the rigid body subject to the nonholonomic constraint $\langle\gamma, \omega\rangle=0$ (Veselova problem), rotating under the action of a force field is defined by the potential

$$
U=-\frac{\Psi_{1}^{2}+\Psi_{2}^{2}}{2\left(I_{1} \gamma_{2}^{2}+I_{2} \gamma_{1}^{2}\right)},
$$

where $\Psi_{j}=\Psi_{j}\left(x, z, K_{3}, K_{4}\right)$ for $j=1,2$ and $K_{3}$ and $K_{4}$ are constants. Assume that $\Psi_{1}$ and $\Psi_{2}$ are solutions of the first order partial differential equation

$$
\begin{aligned}
\Theta:= & \left(I_{1}-I_{2}\right) \sin z \cos z \sin x \cos x \frac{\partial \Psi_{2}}{\partial z} \\
& +\left(I_{1} \cos ^{2} x+I_{2} \sin ^{2} x\right) \frac{\partial \Psi_{2}}{\partial x}-p \sin z \frac{\partial \Psi_{1}}{\partial z}=0
\end{aligned}
$$

satisfying

$$
\frac{\partial \Psi_{1}}{\partial K_{3}} \frac{\partial \Psi_{2}}{\partial K_{4}}-\frac{\partial \Psi_{2}}{\partial K_{3}} \frac{\partial \Psi_{1}}{\partial K_{4}} \neq 0 \quad \text { for all }\left(\gamma_{1}, \gamma_{2}, \gamma_{3}\right) \in \mathbb{R}^{3}
$$

Then the following statements hold.

(a) System (11) has the general integrals

$$
F_{1}=I_{1} \omega_{1} \gamma_{2}-I_{2} \omega_{2} \gamma_{1}-\Psi_{2}=0, \quad F_{2}=p \omega_{3}-\Psi_{1}=0 .
$$

Moreover system (11) is quasi-implicitly integrable. 
(b) If $\Psi_{1}^{2}+\Psi_{2}^{2}=2 \Psi(x)$, then system (11) has the first integral

$$
I_{1} \omega_{1} \gamma_{2}-I_{2} \omega_{2} \gamma_{1}=K_{4} .
$$

Consequently the system is explicitly integrable.

(c) If $I_{1} \neq I_{2}$ and $\Psi_{1}^{2}+\Psi_{2}^{2}=2 \Psi\left(\frac{I_{1} \gamma_{2}^{2}+I_{2} \gamma_{1}^{2}}{\gamma_{3}^{2}}\right)$, then the system has the first integral

$$
\sqrt{I_{1} I_{2} I_{3}\left(\frac{\gamma_{1}^{2}}{I_{1}}+\frac{\gamma_{2}^{2}}{I_{2}}+\frac{\gamma_{3}^{2}}{I_{3}}\right)} \omega_{3}=K_{4}
$$

If $I_{1}=I_{2}$ and $\Psi_{1}^{2}+\Psi_{3}^{2}=2 \Psi^{2}(z)$, then system has the first integral

$$
\sqrt{I_{3}\left(\frac{\gamma_{1}^{2}+\gamma_{2}^{2}}{I_{1}}+\frac{\gamma_{3}^{2}}{I_{3}}\right)} \omega_{3}=K_{4}
$$

Consequently the system is explicitly integrable.

(d) Using (23) the constraint $\gamma_{1} \omega_{1}+\gamma_{2} \omega_{2}+\gamma_{3} \omega_{3}=0$, and the fact that $\gamma_{1}^{2}+$ $\gamma_{2}^{2}+\gamma_{3}^{2}=1$, the Poisson equations for the Veselova problem take the form

$$
\begin{aligned}
& \dot{\gamma}_{1}=-\frac{\gamma_{2}\left(I_{1}+\left(I_{2}-I_{1}\right) \gamma_{1}^{2}\right) \Psi_{1}+\gamma_{3} \gamma_{1} \Psi_{2}}{p\left(I_{2} \gamma_{1}^{2}+I_{2} \gamma_{2}^{2}\right)}, \\
& \dot{\gamma}_{2}=\frac{\left.\gamma_{1}\left(I_{2}+\left(I_{1}-I_{2}\right) \gamma_{2}^{2}\right)\right) \Psi_{1}-\gamma_{3} \gamma_{2} \Psi_{2}}{p\left(I_{2} \gamma_{1}^{2}+I_{2} \gamma_{2}^{2}\right)}, \\
& \dot{\gamma}_{3}=\frac{\gamma_{1} \gamma_{2} \gamma_{3}\left(I_{1}-I_{2}\right) \Psi_{1}+p\left(\gamma_{2}^{2}+\gamma_{1}^{2}\right) \Psi_{2}}{p\left(I_{2} \gamma_{1}^{2}+I_{2} \gamma_{2}^{2}\right)} .
\end{aligned}
$$

We observe that the integral (24) is well known (see for instance [6]).

Corollary 1 Equation (21) is equivalent to the equation

$$
\left(I_{2}-I_{1}\right) \gamma_{1} \gamma_{2}\left\langle\gamma, \frac{\partial \Psi_{2}}{\partial \gamma}\right\rangle+I_{1} \gamma_{2} \frac{\partial \Psi_{2}}{\partial \gamma_{1}}-I_{2} \gamma_{1} \frac{\partial \Psi_{2}}{\partial \gamma_{2}}-p\left(\gamma_{3}\left\langle\gamma, \frac{\partial \Psi_{1}}{\partial \gamma}\right\rangle-\frac{\partial \Psi_{1}}{\partial \gamma_{3}}\right)=0 .
$$

The proof of Theorem 2 and Corollary 1 are given in section 4 .

We note that the potential (20) contains as particular subcases the potential studied by Veselova and Fedorov-Jovanovic [9].

Under the assumption $\Delta=\left(I_{1}-I_{2}\right)\left(I_{2}-I_{3}\right)\left(I_{3}-I_{1}\right) \neq 0$ we introduce the coordinates $\left(\tau_{1}, \tau_{2}, \tau_{3}\right)$ such that

$$
\tau_{1}=\gamma_{1}^{2}+\gamma_{2}^{2}+\gamma_{3}^{2}, \quad \tau_{2}=I_{1} \gamma_{1}^{2}+I_{2} \gamma_{2}^{2}+I_{3} \gamma_{3}^{2}, \quad \tau_{3}=\frac{\gamma_{1}^{2}}{I_{1}}+\frac{\gamma_{2}^{2}}{I_{2}}+\frac{\gamma_{3}^{2}}{I_{3}} .
$$

Hence

$$
\begin{aligned}
& \gamma_{1}^{2}=r_{1}\left(\left(I_{2}+I_{3}\right) \tau_{1}-\tau_{2}-I_{2} I_{3} \tau_{3}\right), \\
& \gamma_{2}^{2}=r_{2}\left(\left(I_{1}+I_{3}\right) \tau_{1}-\tau_{2}-I_{1} I_{3} \tau_{3}\right), \\
& \gamma_{3}^{2}=r_{3}\left(\left(I_{2}+I_{1}\right) \tau_{1}-\tau_{2}-I_{2} I_{1} \tau_{3}\right),
\end{aligned}
$$


where

$$
r_{1}=\frac{\left(I_{2}-I_{3}\right) I_{1}}{\Delta}, \quad r_{2}=\frac{\left(I_{3}-I_{1}\right) I_{2}}{\Delta}, \quad r_{3}=\frac{\left(I_{1}-I_{2}\right) I_{3}}{\Delta}
$$

Our last results on the Veselova problems are the following theorem and corollaries.

Theorem 3 The following statements hold for the Veselova problem.

(a) System (11) for $\Delta \neq 0$ and $\mu \neq 0$ (i.e. we have a constrained system) admits the first integral

$$
\Phi_{4}=\frac{1}{2}\|\gamma \wedge I \omega\|^{2}-W\left(\tau_{2}, \tau_{3}\right)
$$

if and only if the potential function $U$ and the function $W$ satisfy the partial differential equations of first order

$\frac{\partial U}{\partial \tau_{3}}=\tilde{\nu} \tau_{1}, \quad \frac{\partial W}{\partial \tau_{2}}=\tilde{\nu} \tau_{3}, \quad|I| \frac{\partial U}{\partial \tau_{2}}+\frac{\partial W}{\partial \tau_{3}}=\left(-\tau_{2}+\left(I_{1}+I_{2}+I_{3}\right) \tau_{1}\right) \tilde{\nu}$,

where $|I|=I_{1} I_{2} I_{3}$ and $\left(\tau_{1}, \tau_{2}, \tau_{3}\right)$ are the variables defined in (27) and $\tilde{\nu}=\tilde{\nu}\left(\tau_{2}, \tau_{3}\right)$ is an arbitrary function.

(b) System (11) with $\Delta \neq 0$ and $\mu=0$ (i.e. the system has no constraints) admits the first integral (29) if and only if the potential function $U$ and the function $W$ satisfy the following partial differential equations of first order

$$
\frac{\partial U}{\partial \tau_{3}}=0, \quad \frac{\partial W}{\partial \tau_{2}}=0, \quad|I| \frac{\partial U}{\partial \tau_{2}}+\frac{\partial W}{\partial \tau_{3}}=0
$$

Clearly for the real motion $\tau_{1}=1$.

Corollary 2 The following statements hold.

(a) System (11) with $\Delta \neq 0$ and $\mu \neq 0$ admits the first integral $\Phi_{4}$ given by

$$
\begin{aligned}
& \frac{1}{2}\|\gamma \wedge I \omega\|^{2}-\left.\int_{\tau_{2}^{0}}^{\tau_{2}} \tau_{3} \frac{\partial U}{\partial \tau_{3}}\right|_{\tau_{3}=\tau_{3}^{0}} d \tau_{2}+ \\
& \quad \int_{\tau_{3}^{0}}^{\tau_{3}}\left(|I| \frac{\partial U}{\partial \tau_{2}}+\left(\tau_{2}-\left(I_{1}+I_{2}+I_{3}\right)\right) \frac{\partial U}{\partial \tau_{3}}\right) d \tau_{3}
\end{aligned}
$$

if and only if the potential function $U$ satisfies the linear second order partial differential equations

$$
2 \frac{\partial U}{\partial \tau_{3}}+|I| \frac{\partial^{2} U}{\partial \tau_{2}^{2}}+\left(\tau_{2}-\left(I_{1}+I_{2}+I_{3}\right)\right) \frac{\partial^{2} U}{\partial \tau_{3} \partial \tau_{2}}+\tau_{3} \frac{\partial^{2} U}{\partial \tau_{3}^{2}}=0
$$


(b) System (11) with $\Delta \neq 0$ and $\mu=0$ admits the first integral $\Phi_{4}$ given by

$$
\frac{1}{2}\|\gamma \wedge I \omega\|^{2}+\int_{\tau_{3}^{0}}^{\tau_{3}}\left(|I| \frac{\partial U}{\partial \tau_{2}}\right) d \tau_{3}
$$

if and only if the potential function $U$ satisfies the linear second order partial differential equations

$$
\frac{\partial U}{\partial \tau_{3}}=0, \quad \frac{\partial^{2} U}{\partial \tau_{2} \partial \tau_{2}}=0 .
$$

Consequently $U=\alpha \tau_{2}$, where $\alpha$ is a constant, is the Klebsh-Tisserand potential, and $W=-|I| \alpha \tau_{3}$. Thus the first integral $\Phi_{4}$ is

$$
\frac{1}{2}\|\gamma \wedge I \omega\|^{2}+|I| \alpha \tau_{3}
$$

Corollary 3 A particular solution of (33) is the potential function

$$
\begin{aligned}
U= & a_{0}+a_{1} \tau_{2}+a_{2}\left(\tau_{2}^{2}-|I| \tau_{3}\right)+ \\
& \frac{\alpha_{3}}{r_{1}\left(\left(I_{2}+I_{3}\right) \tau_{1}-\tau_{2}-I_{2} I_{3} \tau_{3}\right)}+\frac{\alpha_{4}}{r_{2}\left(\left(I_{1}+I_{3}\right) \tau_{1}-\tau_{2}-I_{1} I_{3} \tau_{3}\right)}+ \\
& \frac{\alpha_{5}}{r_{3}\left(\left(I_{2}+I_{1}\right) \tau_{1}-\tau_{2}-I_{2} I_{1} \tau_{3}\right)},
\end{aligned}
$$

where $a_{0}$ and $\alpha_{j}$ for $j=3,4,5$ are constants. Consequently we have the first integral

$$
\begin{aligned}
\Phi_{4}= & \frac{1}{2}\|\gamma \wedge I \omega\|^{2}-|I| a_{2}\left(\tau_{2} \tau_{3}+\left(I_{1}+I_{2}+I_{3}\right) \tau_{3}\right)+|I| a_{1} \tau_{3}+ \\
& \frac{\alpha_{3}\left(\tau_{2}-I_{2}-I_{3}\right)}{r_{1}\left(\left(I_{2}+I_{3}\right) \tau_{1}-\tau_{2}-I_{2} I_{3} \tau_{3}\right)}+\frac{\alpha_{4}\left(\tau_{2}-I_{1}-I_{3}\right)}{r_{2}\left(\left(I_{1}+I_{3}\right) \tau_{1}-\tau_{2}-I_{1} I_{3} \tau_{3}\right)}+ \\
& \frac{\alpha_{5}\left(\tau_{2}-I_{2}-I_{1}\right)}{r_{3}\left(\left(I_{2}+I_{1}\right) \tau_{1}-\tau_{2}-I_{2} I_{1} \tau_{3}\right)}
\end{aligned}
$$

The proof of Theorem 3 and Corollaries 2 and 3 are given in section 5 .

Remark 4 Fedorov and Jovanovic in [9] claimed that

$$
\begin{aligned}
\Phi= & \frac{1}{2}\|\gamma \wedge I \omega\|^{2}-W\left(\tau_{2}, \tau_{3}\right) \\
& =\frac{1}{2}\|\gamma \wedge I \omega\|^{2}+\alpha_{1}|I|\langle I \gamma, \gamma\rangle\left\langle I^{-1} \gamma, \gamma\right\rangle-\alpha_{2}|I|\left\langle I^{-1} \gamma, \gamma\right\rangle \\
& +\alpha_{3}\left(I_{2} \frac{\gamma_{2}^{2}}{\gamma_{1}^{2}}+I_{3} \frac{\gamma_{3}^{2}}{\gamma_{1}^{2}}\right)+\alpha_{4}\left(I_{3} \frac{\gamma_{3}^{2}}{\gamma_{2}^{2}}+I_{1} \frac{\gamma_{1}^{2}}{\gamma_{2}^{2}}\right)+\alpha_{5}\left(I_{1} \frac{\gamma_{1}^{2}}{\gamma_{3}^{2}}+I_{2} \frac{\gamma_{2}^{2}}{\gamma_{3}^{2}}\right)
\end{aligned}
$$

is a first integral of system (11) with the potential

$$
U=\alpha_{1}\left(\left\langle I^{2} \gamma, \gamma\right\rangle-\langle I \gamma, \gamma\rangle^{2}\right)+\alpha_{2}\langle I \gamma, \gamma\rangle+\frac{\alpha_{3}}{\gamma_{1}^{2}}+\frac{\alpha_{4}}{\gamma_{2}^{2}}+\frac{\alpha_{5}}{\gamma_{3}^{2}}
$$


where $\alpha_{j}$ for $j=1, \ldots, 5$ are constants.

In fact the first integral (36) of system (11) for the previous potential in the variables $\left(\tau_{1}, \tau_{2}, \tau_{3}\right)$ (see $\left.(28)\right)$ becomes

$$
\begin{aligned}
\Phi= & \frac{1}{2}\|\gamma \wedge I \omega\|^{2}-W\left(\tau_{2}, \tau_{3}\right) \\
= & \frac{1}{2}\|\gamma \wedge I \omega\|^{2}+\alpha_{1}|I| \tau_{2} \tau_{3}-\alpha_{2}|I| \tau_{3} \\
& \frac{\alpha_{3} \tau_{2}}{r_{1}\left(\left(I_{2}+I_{3}\right) \tau_{1}-\tau_{2}-I_{2} I_{3} \tau_{3}\right)}+\frac{\alpha_{4} \tau_{2}}{r_{2}\left(\left(I_{1}+I_{3}\right) \tau_{1}-\tau_{2}-I_{1} I_{3} \tau_{3}\right)}+ \\
& \frac{\alpha_{5} \tau_{2}}{r_{3}\left(\left(I_{2}+I_{1}\right) \tau_{1}-\tau_{2}-I_{2} I_{1} \tau_{3}\right)}-\alpha_{3} I_{1}-\alpha_{4} I_{2}-\alpha_{5} I_{3},
\end{aligned}
$$

The potential (37) and the function $W$ defined in (36) write in the variables $\tau_{2}$ and $\tau_{3}$ as

$$
\begin{aligned}
U= & \alpha_{1}\left(\left(I_{1}+I_{2}+I_{3}\right) \tau_{2}+|I| \tau_{3}-\left(I_{1} I_{2}+I_{2} I_{3}+I_{3} I_{1}\right)-\tau_{2}^{2}\right)+\alpha_{2} \tau_{2}+ \\
& \frac{\alpha_{3}}{r_{1}\left(\left(I_{2}+I_{3}\right) \tau_{1}-\tau_{2}-I_{2} I_{3} \tau_{3}\right)}+\frac{\alpha_{4}}{r_{2}\left(\left(I_{1}+I_{3}\right) \tau_{1}-\tau_{2}-I_{1} I_{3} \tau_{3}\right)}+ \\
& \frac{\alpha_{5}}{r_{3}\left(\left(I_{2}+I_{1}\right) \tau_{1}-\tau_{2}-I_{2} I_{1} \tau_{3}\right)}, \\
W= & \alpha_{1}|I| \tau_{2} \tau_{3}-\alpha_{2}|I| \tau_{3} \\
& \frac{\alpha_{3} \tau_{2}}{r_{1}\left(\left(I_{2}+I_{3}\right) \tau_{1}-\tau_{2}-I_{2} I_{3} \tau_{3}\right)}+\frac{\alpha_{4} \tau_{2}}{r_{2}\left(\left(I_{1}+I_{3}\right) \tau_{1}-\tau_{2}-I_{1} I_{3} \tau_{3}\right)}+ \\
& \frac{\alpha_{5} \tau_{2}}{r_{3}\left(\left(I_{2}+I_{1}\right) \tau_{1}-\tau_{2}-I_{2} I_{1} \tau_{3}\right)}-\alpha_{3} I_{1}-\alpha_{4} I_{2}-\alpha_{5} I_{3} .
\end{aligned}
$$

Note that $U$ coincide with the potential (35). Since $W$ does not satisfy (30) with $U$ given by (38), it follows that $\Phi$ is not a first integral. 


\section{Proof of Theorem 1}

After some calculations we obtain that the derivative of $F_{1}$ along the solutions of (9) takes the form

$$
\begin{aligned}
\dot{F}_{1}= & I_{1} \dot{\omega}_{1}-\dot{\mu}_{2} \\
= & \gamma_{2} \frac{\partial U}{\partial \gamma_{3}}-\gamma_{3} \frac{\partial U}{\partial \gamma_{2}}+\frac{\partial \mu_{2}}{\partial \gamma_{1}} \gamma_{3} \omega_{2}-\frac{\partial \mu_{2}}{\partial \gamma_{2}} \gamma_{3} \omega_{1}-\frac{\partial \mu_{2}}{\partial \gamma_{3}}\left(\gamma_{1} \omega_{2}-\gamma_{2} \omega_{1}\right) \\
& -\frac{\partial \mu_{2}}{\partial K_{1}} \dot{K}_{1}-\frac{\partial \mu_{2}}{\partial K_{2}} \dot{K}_{2} \\
= & \gamma_{2} \frac{\partial U}{\partial \gamma_{3}}-\gamma_{3} \frac{\partial U}{\partial \gamma_{2}}+\omega_{2}\left(-\gamma_{1} \frac{\partial \mu_{2}}{\partial \gamma_{3}}+\gamma_{3} \frac{\partial \mu_{2}}{\partial \gamma_{1}}\right)+\omega_{1}\left(-\gamma_{3} \frac{\partial \mu_{2}}{\partial \gamma_{2}}+\gamma_{2} \frac{\partial \mu_{2}}{\partial \gamma_{3}}\right) \\
= & \gamma_{2} \frac{\partial U}{\partial \gamma_{3}}-\gamma_{3} \frac{\partial U}{\partial \gamma_{2}}+\frac{F_{2}-\mu_{1}}{I_{2}}\left(-\gamma_{1} \frac{\partial \mu_{2}}{\partial \gamma_{3}} \gamma_{3} \frac{\partial \mu_{2}}{\partial \gamma_{1}}\right)+ \\
& +\frac{F_{1}+\mu_{2}}{I_{1}}\left(-\gamma_{3} \frac{\partial \mu_{2}}{\partial \gamma_{2}}+\gamma_{2} \frac{\partial \mu_{2}}{\partial \gamma_{3}}\right) \\
= & \gamma_{2} \frac{\partial}{\partial \gamma_{3}}\left(U+\frac{1}{2 I_{1} I_{2}}\left(I_{1} \mu_{1}^{2}+I_{2} \mu_{2}^{2}\right)\right)-\gamma_{3} \frac{\partial}{\partial \gamma_{2}}\left(U+\frac{1}{2 I_{1} I_{2}}\left(I_{1} \mu_{1}^{2}+I_{2} \mu_{2}^{2}\right)\right) \\
& +\frac{\mu_{1}}{I_{2}}\left(\gamma_{3}\left(\frac{\partial \mu_{1}}{\partial \gamma_{2}}-\frac{\partial \mu_{2}}{\partial \gamma_{1}}\right)-\gamma_{2} \frac{\partial \mu_{1}}{\partial \gamma_{3}}+\gamma_{1} \frac{\partial \mu_{2}}{\partial \gamma_{3}}\right) \\
& -\frac{F_{2}}{I_{2}}\left(\gamma_{1} \frac{\partial \mu_{2}}{\partial \gamma_{3}}-\gamma_{3} \frac{\partial \mu_{2}}{\partial \gamma_{1}}\right)-\frac{F_{1}}{I_{1}}\left(\gamma_{3} \frac{\partial \mu_{2}}{\partial \gamma_{2}}-\gamma_{2} \frac{\partial \mu_{2}}{\partial \gamma_{3}}\right)
\end{aligned}
$$

here we use that $\dot{K}_{1}=\dot{K}_{2}=0$.

In view of (14) and (15) we obtain

$$
\dot{F}_{1}=\frac{F_{2}}{I_{2}}\left(\gamma_{1} \frac{\partial \mu_{2}}{\partial \gamma_{3}}-\gamma_{3} \frac{\partial \mu_{2}}{\partial \gamma_{1}}\right)+\frac{F_{1}}{I_{1}}\left(\gamma_{3} \frac{\partial \mu_{2}}{\partial \gamma_{2}}-\gamma_{2} \frac{\partial \mu_{2}}{\partial \gamma_{3}}\right)
$$

A similar relation can obtained for $\dot{F}_{2}$. Hence, by considering (17) we deduce that $\left.\dot{F}_{j}\right|_{F_{1}=F_{2}=0}=0$ for $j=1,2$. Therefore $F_{1}=0$ and $F_{2}=0$ are two general integrals. Consequently there are two independent local first integrals

$$
\Phi_{1}\left(\omega_{1}, \omega_{2}, \gamma_{1}, \gamma_{2}, \gamma_{3}\right)=K_{3}, \quad \Phi_{4}\left(\omega_{1}, \omega_{2}, \gamma_{1}, \gamma_{2}, \gamma_{3}\right)=K_{4}
$$

Thus system (9) is locally explicitly integrable. We have a third general integral

$$
\left.F_{3}\left(\gamma_{1}, \gamma_{2}, \gamma_{3}, K_{3}\right)\right|_{K_{3}=1}=\gamma_{1}^{2}+\gamma_{2}^{2}+\gamma_{3}^{2}-1=0 .
$$

On the other hand, system (9) has divergence zero due to the fact that its flow preserves the Lebesgue measure, see Proposition 1.

In short, by the Euler-Jacobi Theorem it follows that system (9) is quasiimplicitly integrable. Hence statement (a) is proved. 
From Remark 2 first we consider the functions

$$
\mu_{1}=\frac{\partial \tilde{S}\left(\gamma_{1}, \gamma_{2}, \gamma_{3}, K_{1}, K_{4}\right)}{\partial \gamma_{1}}=\frac{\partial \tilde{S}}{\partial \gamma_{1}}, \quad \mu_{2}=\frac{\partial \tilde{S}\left(\gamma_{1}, \gamma_{2}, \gamma_{3}, K_{1}, K_{4}\right)}{\partial \gamma_{2}}=\frac{\partial \tilde{S}}{\partial \gamma_{2}} .
$$

Hence the equations (17) become

$$
F_{1}=I_{1} \omega_{1}-\frac{\partial \tilde{S}}{\partial \gamma_{2}}=0, \quad F_{2}=I_{2} \omega_{2}+\frac{\partial \tilde{S}}{\partial \gamma_{1}}=0
$$

Now we show that the Suslov's, Kharlamova-Zabelina's and Kozlov's first integral can be obtained from (39) .

For the Suslov integrable case $\left(U=\mathrm{constant}\right.$, and $\left.I_{1} \neq I_{2}\right)$ we have that $\tilde{S}=C_{1} \gamma_{1}+C_{2} \gamma_{2}$, where $C_{1}=C_{1}\left(K_{3}, K_{4}\right)$ and $C_{2}=C_{2}\left(K_{3}, K_{4}\right)$ are arbitrary constants. Since $\mu_{1}=C_{1}$ and $\mu_{2}=C_{2}$, taking

$$
C_{1}=\sqrt{\frac{I_{2}\left(I_{1} K_{3}-K_{4}\right)}{I_{1}-I_{2}}}, \quad C_{2}=\sqrt{\frac{I_{1}\left(K_{4}-I_{2} K_{3}\right)}{I_{1}-I_{2}}},
$$

(16) holds. Equations (17) write

$$
F_{1}=I_{1} \omega_{1}-C_{2}=0, \quad F_{2}=I_{2} \omega_{2}+C_{1}=0 .
$$

By solving this system with respect to $K_{3}$ and $K_{4}$ we obtain

$$
K_{3}=I_{1} \omega_{1}^{2}+I_{2} \omega_{2}^{2}=\frac{C_{2}^{2}}{I_{2}}+\frac{C_{1}^{2}}{I_{1}}, \quad K_{4}=I_{1}^{2} \omega_{1}^{2}+I_{2}^{2} \omega_{2}^{2}=C_{2}^{2}+C_{1}^{2} .
$$

Note that $K_{3}$ is the energy first integral, and $K_{4}$ is the kinetic moment (the Suslov first integral).

For the Kharlamova-Zabelina integrable case $(U=\langle\mathbf{b}, \gamma\rangle)$ we take the function $\tilde{S}$ of the Remark 2 as

$$
\tilde{S}=\frac{2 / 3}{\sqrt{I_{1} b_{1}^{2}+I_{2} b_{2}^{2}}}\left(\tilde{h}+b_{1} \gamma_{1}+b_{2} \gamma_{2}\right)^{3 / 2}-\frac{K_{4}}{b_{1}^{2} I_{1}+b_{2}^{2} I_{2}}\left(b_{2} I_{2} \gamma_{1}-b_{1} I_{1} \gamma_{2}\right)
$$

where $\tilde{h}=I_{1} I_{2}\left(\frac{K_{4}^{2} I_{1} I_{2}}{b_{1}^{2} I_{1}+b_{2}^{2} I_{2}}-K_{3}\right), K_{3}$ and $K_{4}$ are arbitrary constants. Then

$$
\begin{aligned}
& \mu_{1}=\frac{b_{1}}{\sqrt{I_{1} b_{1}^{2}+I_{2} b_{2}^{2}}} \sqrt{\tilde{h}+b_{1} \gamma_{1}+b_{2} \gamma_{2}}-\frac{K_{4} b_{2} I_{2}}{b_{1}^{2} I_{1}+b_{2}^{2} I_{2}}, \\
& \mu_{2}=\frac{b_{2}}{\sqrt{I_{1} b_{1}^{2}+I_{2} b_{2}^{2}}} \sqrt{\tilde{h}+b_{1} \gamma_{1}+b_{2} \gamma_{2}}+\frac{K_{4} b_{1} I_{1}}{b_{1}^{2} I_{1}+b_{2}^{2} I_{2}} .
\end{aligned}
$$

Therefore equations (17) take the form

$$
\begin{aligned}
& F_{1}=I_{1} \omega_{1}-\left(\frac{b_{2}}{\sqrt{I_{1} b_{1}^{2}+I_{2} b_{2}^{2}}} \sqrt{\tilde{h}+b_{1} \gamma_{1}+b_{2} \gamma_{2}}+\frac{K_{4} b_{1} I_{1}}{b_{1}^{2} I_{1}+b_{2}^{2} I_{2}}\right)=0 \\
& F_{2}=I_{2} \omega_{2}+\left(\frac{b_{1}}{\sqrt{I_{1} b_{1}^{2}+I_{2} b_{2}^{2}}} \sqrt{\tilde{h}+b_{1} \gamma_{1}+b_{2} \gamma_{2}}-\frac{K_{4} b_{2} I_{2}}{b_{1}^{2} I_{1}+b_{2}^{2} I_{2}}\right)=0 .
\end{aligned}
$$


Solving this system with respect to $K_{3}$ and $K_{4}$ we obtain

$$
K_{3}=I_{1} \omega_{1}^{2}+I_{2} \omega_{2}^{2}-\frac{1}{I_{1} I_{2}}\left(b_{1} \gamma_{1}+b_{2} \gamma_{2}\right), \quad K_{4}=I_{1} \omega_{1} b_{1}+I_{2} \omega_{2} b_{2} .
$$

Again $K_{3}$ is the energy integral and $K_{4}$ is the well-known Kharlamova-Zabelina first integral [11].

For the Kozlov's integrable case $\left(U=a \gamma_{3}\right.$ and $\left.I_{1}=I_{2}\right)$ we take

$$
\tilde{S}=-K_{4} \arctan \left(\frac{\gamma_{1}}{\gamma_{2}}\right)+\frac{1}{2} \int D\left(\gamma_{1}^{2}+\gamma_{2}^{2}\right) d\left(\gamma_{1}^{2}+\gamma_{2}^{2}\right),
$$

where

$$
D(u)=I_{1} \sqrt{\frac{K_{3}+a \sqrt{1-u}}{u}-\frac{K_{4}^{2}}{u^{2}}},
$$

$a, \quad K_{3}$ and $K_{4}$ are constants. Hence

$$
\mu_{1}=-\frac{\gamma_{2} K_{4}}{\gamma_{1}^{2}+\gamma_{2}^{2}}+\gamma_{1} D\left(\gamma_{1}^{2}+\gamma_{2}^{2}\right), \quad \mu_{2}=\frac{\gamma_{1} K_{4}}{\gamma_{1}^{2}+\gamma_{2}^{2}}+\gamma_{2} D\left(\gamma_{1}^{2}+\gamma_{2}^{2}\right) .
$$

Consequently the functions $F_{1}$ and $F_{2}$ of (17) are

$$
\begin{aligned}
& F_{1}=\omega_{1}-\left(\frac{\gamma_{1} K_{4}}{\gamma_{1}^{2}+\gamma_{2}^{2}}+\gamma_{2} D\left(\gamma_{1}^{2}+\gamma_{2}^{2}\right)=0,\right. \\
& F_{2}=\omega_{2}+\left(-\frac{\gamma_{2} K_{4}}{\gamma_{1}^{2}+\gamma_{2}^{2}}+\gamma_{1} D\left(\gamma_{1}^{2}+\gamma_{2}^{2}\right)=0 .\right.
\end{aligned}
$$

Therefore, by solving this system with respect to $K_{3}$ and $K_{4}$ we obtain

$$
K_{3}=\omega_{1}^{2}+\omega_{2}^{2}-a \sqrt{1-\gamma_{1}^{2}-\gamma_{2}^{2}}=\omega_{1}^{2}+\omega_{2}^{2}-a \gamma_{3}, \quad K_{4}=\omega_{1} \gamma_{1}+\omega_{2} \gamma_{2} .
$$

So $K_{3}$ is the energy integral and $K_{4}$ is the Kozlov-Lagrange first integral. In fact, this integral correspond to the well-known integrable "Lagrange case" of the Suslov problem [13].

Finally we analyze the case when the functions $\mu_{1}$ and $\mu_{2}$ of (19) are given by the formula

$$
\mu_{1}=\Psi_{1}\left(\gamma_{1}^{2}+\gamma_{3}^{2}, \gamma_{1}, K_{3}, K_{4}\right), \quad \mu_{2}=\Psi_{2}\left(\gamma_{1}^{2}+\gamma_{3}^{2}, \gamma_{2}, K_{3}, K_{4}\right) .
$$

We observe that these solutions, obtained integrating the system

$$
\gamma_{3} \frac{\partial \mu_{1}}{\partial \gamma_{2}}-\gamma_{2} \frac{\partial \mu_{1}}{\partial \gamma_{3}}=0, \quad \gamma_{3} \frac{\partial \mu_{2}}{\partial \gamma_{1}}-\gamma_{1} \frac{\partial \mu_{2}}{\partial \gamma_{3}}=0,
$$

are a particular case of equations (15). The potential function (14) in this case coincides with the potential obtained by Dragović-Gajić-Jovanović in [6]. We call this case the Generalized Tisserand case. In particular, if $I_{1} \neq I_{2}$ and

$$
\begin{aligned}
& \mu_{1}=\sqrt{h_{1}+\left(a_{1}+a_{3}\right)\left(\gamma_{3}^{2}+\gamma_{2}^{2}\right)+\left(b_{1}+a_{3}\right) \gamma_{1}^{2}+f_{1}\left(\gamma_{1}\right)}, \\
& \mu_{2}=\sqrt{h_{2}+\left(a_{2}+a_{4}\right)\left(\gamma_{3}^{2}+\gamma_{1}^{2}\right)+\left(b_{2}+a_{4}\right) \gamma_{2}^{2}+f_{2}\left(\gamma_{2}\right)},
\end{aligned}
$$


where $a_{1}, a_{2}, a_{3}, a_{4}, b_{1}, b_{2}$ and

$$
h_{1}=\frac{I_{2}\left(I_{1} K_{3}-K_{4}\right)}{I_{1}-I_{2}}, \quad h_{2}=\frac{I_{1}\left(I_{2} K_{3}-K_{4}\right)}{I_{1}-I_{2}}
$$

are constants, and $f_{1}$ and $f_{2}$ are arbitrary functions, then the general integrals $F_{1}=0$ and $F_{2}=0$ take the form

$$
\begin{aligned}
& F_{1}=I_{1} \omega_{1}-\sqrt{h_{2}+\left(a_{2}+a_{4}\right)\left(\gamma_{3}^{2}+\gamma_{1}^{2}\right)+\left(b_{2}+a_{4}\right) \gamma_{2}^{2}+f_{2}\left(\gamma_{2}\right)}=0 \\
& F_{2}=I_{2} \omega_{2}+\sqrt{h_{1}+\left(a_{1}+a_{3}\right)\left(\gamma_{3}^{2}+\gamma_{2}^{2}\right)+\left(b_{1}+a_{3}\right) \gamma_{1}^{2}+f_{1}\left(\gamma_{1}\right)}=0 .
\end{aligned}
$$

The case when $f_{j}\left(\gamma_{j}\right)=\alpha_{j} \gamma_{j}$, for $j=1,2$ was studied by Okuneva in [17], where $\alpha_{1}$ and $\alpha_{2}$ are constants.

If $f_{1}=f_{2}=0$, we obtain the Tisserand's case [12]. By solving $F_{j}=0$ for $j=1,2$ with respect to $K_{3}$ and $K_{4}$ we get that the first integrals in the Klebsh-Tisserand's case are

$$
\begin{aligned}
K_{3}= & I_{1} \omega_{1}^{2}+I_{2} \omega_{2}^{2}-\left(\frac{b_{1}+a_{3}}{I_{2}}+\frac{a_{2}+a_{4}}{I_{1}}\right) \gamma_{1}^{2} \\
& -\left(\frac{a_{1}+a_{3}}{I_{2}}+\frac{b_{2}+a_{4}}{I_{1}}\right) \gamma_{2}^{2}-\left(\frac{a_{1}+a_{3}}{I_{2}}+\frac{a_{2}+a_{4}}{I_{1}}\right) \gamma_{3}^{2}, \\
K_{4}= & I_{1}^{2} \omega_{1}^{2}+I_{2}^{2} \omega_{2}^{2}-\left(b_{1}+a_{3}+a_{2}+a_{4}\right) \gamma_{1}^{2} \\
& -\left(a_{1}+a_{3}+b_{2}+a_{4}\right) \gamma_{2}^{2}-\left(a_{1}+a_{3}+a_{2}+a_{4}\right) \gamma_{3}^{2} .
\end{aligned}
$$

In short, statement (b) is proved.

Now we prove statement (c). First we introduce the following well-known definition. An elliptic integral is any integral which can be expressed in the form

$$
\int R(x, \sqrt{P(x)}) d x
$$

where $\mathrm{R}$ is a rational function of its two arguments, $\mathrm{P}$ is a polynomial of degree 3 or 4 with no repeated roots. In general, elliptic integrals cannot be expressed in terms of elementary functions. Exceptions to this general rule are when $\mathrm{P}$ has repeated roots, or when $R(x, y)$ contains no odd powers of $\mathrm{y}$. However, with the appropriate reduction formula, every elliptic integral can be brought into a form that involves integrals over rational functions and the three Legendre canonical forms (i.e. the elliptic integrals of the first, second and third kind).

We observe that from the general integrals (17) it follows that

$$
\omega_{1}=\frac{\mu_{2}}{I_{1}}, \quad \omega_{2}=-\frac{\mu_{1}}{I_{2}},
$$

thus by inserting in the Poisson equation (see formula (9)) we easily obtain (18). 
Now we deal with the case $\mu_{j}=\frac{\partial \tilde{S}\left(\gamma_{1}, \gamma_{2}, \gamma_{3}, K_{3}, K_{4}\right)}{\partial \gamma_{j}}$ for $j=1,2$. Therefore equations (18) becomes

$$
\dot{\gamma}_{1}=-\frac{\gamma_{3}}{I_{2}} \frac{\partial \tilde{S}}{\partial \gamma_{1}}, \quad \dot{\gamma}_{2}=-\frac{\gamma_{3}}{I_{1}} \frac{\partial \tilde{S}}{\partial \gamma_{2}}, \quad \dot{\gamma}_{3}=\frac{\gamma_{1}}{I_{2}} \frac{\partial \tilde{S}}{\partial \gamma_{1}}+\frac{\gamma_{2}}{I_{1}} \frac{\partial \tilde{S}}{\partial \gamma_{2}} .
$$

The vector $\gamma=\gamma(t)$ is determined integrating system (42).

For the Suslov case the differential system (42) takes the form

$$
\dot{\gamma}_{1}=-\frac{\gamma_{3} C_{1}}{I_{2}}, \quad \dot{\gamma}_{2}=-\frac{\gamma_{3} C_{2}}{I_{1}}, \quad \dot{\gamma}_{3}=\frac{\gamma_{1} C_{1}}{I_{2}}+\frac{\gamma_{2} C_{2}}{I_{1}} .
$$

After integration and taking into account that $\gamma_{1}^{2}+\gamma_{2}^{2}+\gamma_{3}^{2}=1$ we deduce that

$$
\begin{aligned}
& \gamma_{1}(t)=\frac{C_{1} I_{1}}{\sqrt{I_{1}^{2} C_{1}^{2}+I_{2}^{2} C_{2}^{2}}} \sin \beta \sin \left(\frac{\sqrt{I_{1}^{2} C_{1}^{2}+I_{2}^{2} C_{2}^{2}}}{I_{1} I_{2}} t+\alpha\right)+\frac{I_{2} C_{2} \cos \beta}{\sqrt{I_{1}^{2} C_{1}^{2}+I_{2}^{2} C_{2}^{2}}}, \\
& \gamma_{2}(t)=\frac{C_{2} I_{2}}{\sqrt{I_{1}^{2} C_{1}^{2}+I_{2}^{2} C_{2}^{2}}} \sin \beta \sin \left(\frac{\sqrt{I_{1}^{2} C_{1}^{2}+I_{2}^{2} C_{2}^{2}}}{I_{1} I_{2}} t+\alpha\right)-\frac{I_{1} C_{1} \cos \beta}{\sqrt{I_{1}^{2} C_{1}^{2}+I_{2}^{2} C_{2}^{2}}}, \\
& \gamma_{3}(t)=\sin \beta \cos \left(\sqrt{\frac{I_{1}^{2} C_{1}^{2}+I_{2}^{2} C_{2}^{2}}{I_{1} I_{2}}} t+\alpha\right),
\end{aligned}
$$

where $\alpha, \beta, C_{1}, C_{2}$ are constants.

For the Kharlamova-Zabelina case the differential system (18) is

$$
\begin{aligned}
\dot{\gamma}_{1} & =\frac{\gamma_{3}}{I_{2}}\left(\frac{C_{1}}{\sqrt{I_{1} C_{1}^{2}+I_{2} C_{2}^{2}}} \sqrt{\tilde{h}+C_{1} \gamma_{1}+C_{2} \gamma_{2}}-\frac{K_{4} C_{2} I_{2}}{C_{1}^{2} I_{1}+C_{2}^{2} I_{2}}\right), \\
\dot{\gamma}_{2} & =\frac{\gamma_{3}}{I_{1}}\left(\frac{C_{2}}{\sqrt{I_{1} C_{1}^{2}+I_{2} C_{2}^{2}}} \sqrt{\tilde{h}+C_{1} \gamma_{1}+C_{2} \gamma_{2}}+\frac{K_{4} C_{1} I_{1}}{C_{1}^{2} I_{1}+C_{2}^{2} I_{2}}\right), \\
\dot{\gamma}_{3} & =-\frac{\gamma_{1}}{I_{2}}\left(\frac{C_{1}}{\sqrt{I_{1} C_{1}^{2}+I_{2} C_{2}^{2}}} \sqrt{\tilde{h}+C_{1} \gamma_{1}+C_{2} \gamma_{2}}-\frac{K_{4} C_{2} I_{2}}{C_{1}^{2} I_{1}+C_{2}^{2} I_{2}}\right) \\
& -\frac{\gamma_{2}}{I_{1}}\left(\frac{C_{2}}{\sqrt{I_{1} C_{1}^{2}+I_{2} C_{2}^{2}}} \sqrt{\tilde{h}+C_{1} \gamma_{1}+C_{2} \gamma_{2}}+\frac{K_{4} C_{1} I_{1}}{C_{1}^{2} I_{1}+C_{2}^{2} I_{2}}\right),
\end{aligned}
$$

The solutions of this system are

$$
\begin{aligned}
& \gamma_{1}=\frac{C_{1}}{I_{2}}\left(\tau-\tau_{0}\right)^{2}-\frac{K_{4}}{C_{1}^{2} I_{1}+C_{2}^{2} I_{2}}\left(\tau-\tau_{1}\right), \\
& \gamma_{2}=\frac{C_{2}}{I_{1}}\left(\tau-\tau_{0}\right)^{2}-\frac{K_{4}}{C_{1}^{2} I_{1}+C_{2}^{2} I_{2}}\left(\tau-\tau_{2}\right), \\
& \gamma_{3}=\sqrt{1-\gamma_{1}^{2}-\gamma_{2}^{2}}:=\sqrt{P_{4}\left(\tau, \tau_{0}, \tau_{1}, \tau_{2}, K_{4}\right)}, \\
& t=t_{0}+I_{1} I_{2} \int \frac{d \tau}{\sqrt{P_{4}\left(\tau, \tau_{0}, \tau_{1}, \tau_{2}, K_{4}\right)}},
\end{aligned}
$$


where $\tau_{0}, \tau_{1}, \tau_{2}, K_{4}$ are constants. Note that $P_{4}$ is a polynomial of degree four in the variable $\tau$. Clearly, the equations of motion are integrable in elliptical functions of the time.

The differential system (42) in the Kozlov case takes the form

$\dot{\gamma}_{1}=\gamma_{3}\left(\gamma_{1} D-\frac{\gamma_{2}-K_{4}}{1-\gamma_{3}^{2}}\right), \quad \dot{\gamma}_{2}=\gamma_{3}\left(\gamma_{2} D+\frac{\gamma_{1}-K_{4}}{1-\gamma_{3}^{2}}\right), \quad \dot{\gamma}_{3}=-\left(1-\gamma_{3}^{2}\right) D$

where $D=D\left(1-\gamma_{3}^{2}\right)=D\left(\sin ^{2} z\right)$, and $K_{4}$ is an arbitrary constant. Hence, by considering the constraint $\omega_{3}=\dot{x}+\cos z \dot{y}=0$ we deduce the differential equations

$$
\dot{x}=-\frac{K_{4} \cos z}{\sin ^{2} z}, \quad \dot{y}=\frac{K_{4}}{\sin ^{2} z}, \quad \dot{z}=D\left(\sin ^{2} z\right) \sin z:=-\frac{\sqrt{P_{3}\left(\gamma_{3}, K_{3}, K_{4}, a\right)}}{\sin z},
$$

which are easy to integrate. Note that $P_{3}$ is a polynomial of degree three in the variable $\gamma_{3}$. The solutions are

$$
\begin{aligned}
& x=x_{0}-K_{4} \int \frac{\gamma_{3} d z}{\sin ^{3} z D\left(\sin ^{2} z\right)}=x_{0}+K_{4} \int \frac{\gamma_{3} d \gamma_{3}}{\left(1-\gamma_{3}^{2}\right) \sqrt{P_{3}\left(\gamma_{3}, K_{3}, K_{4}\right)}}, \\
& y=y_{0}+K_{4} \int \frac{d z}{\sin ^{3} z D\left(\sin ^{2} z\right)}=y_{0}-K_{4} \int \frac{d \gamma_{3}}{\left(1-\gamma_{3}^{2}\right) \sqrt{P_{3}\left(\gamma_{3}, K_{3}, K_{4}\right)}}, \\
& t=t_{0}+I_{1} I_{2} \int \frac{d \gamma_{3}}{\sqrt{P_{3}\left(\gamma_{3}, K_{3}, K_{4}\right)}} .
\end{aligned}
$$

As we can observe in the Kozlov case as well as in Lagrange's classical problem of a heavy symmetric top $x, y$ and $t$ are expressed as elliptic integral of $\gamma_{3}$.

For the Generalized Tisserand's case the dependence $\gamma=\gamma(t)$ is determined as follows. Let $\Gamma_{1}$ and $\Gamma_{2}$ be the following functions

$$
\begin{aligned}
& \Gamma_{1}=\Gamma_{1}\left(\gamma_{1}\right)=\left.\Psi_{1}\left(\gamma_{2}^{2}+\gamma_{3}^{2}, \gamma_{1}\right)\right|_{\gamma_{2}^{2}+\gamma_{3}^{2}=1-\gamma_{1}^{2}}, \\
& \Gamma_{2}=\Gamma_{2}\left(\gamma_{2}\right)=\left.\Psi_{2}\left(\gamma_{1}^{2}+\gamma_{3}^{2}, \gamma_{2}\right)\right|_{\gamma_{1}^{2}+\gamma_{3}^{2}=1-\gamma_{2}^{2}}
\end{aligned}
$$

Then the Poisson equations (18) take the form

$$
\dot{\gamma}_{1}=\frac{\gamma_{3}}{I_{2}} \Gamma_{1}, \quad \dot{\gamma}_{2}=\frac{\gamma_{3}}{I_{1}} \Gamma_{2}, \quad \dot{\gamma}_{3}=-\frac{\gamma_{1} \Gamma_{1}}{I_{2}}-\frac{\gamma_{2} \Gamma_{2}}{I_{2}}
$$

The vector $\gamma$ can be obtained as a function of time through the following quadratures

$$
\begin{aligned}
& \int \frac{d \gamma_{1}}{\Gamma_{1}\left(\gamma_{1}\right)}=I_{1}\left(\tau-\tau_{0}\right), \quad \int \frac{d \gamma_{2}}{\Gamma_{2}\left(\gamma_{2}\right)}=I_{2}\left(\tau-\tau_{0}\right) \\
& \sqrt{1-\gamma_{1}^{2}(\tau)-\gamma_{2}^{2}(\tau)}=\gamma_{3}, \quad \int \frac{d \tau}{\sqrt{1-\gamma_{1}^{2}(\tau)-\gamma_{2}^{2}(\tau)}}=\frac{t-t_{0}}{I_{1} I_{2}}
\end{aligned}
$$


For the Tisserand case, if we suppose that $h_{1}+a_{1}+a_{3}>0, a_{1}-b_{1}>0$ and $h_{2}+a_{2}+a_{4}>0, a_{2}-b_{2}>0$, after the integration of equation (44) we obtain

$$
\begin{aligned}
& \gamma_{1}=\sqrt{\frac{h_{1}+a_{1}+a_{3}}{a_{1}-b_{1}}} \sin \left(\sqrt{a_{1}-b_{1}} I_{1}\left(\tau-\tau_{1}\right)\right)=\gamma_{1}(\tau), \\
& \gamma_{2}=\sqrt{\frac{h_{2}+a_{2}+a_{4}}{a_{2}-b_{2}}} \sin \left(\sqrt{a_{2}-b_{2}} I_{2}\left(\tau-\tau_{2}\right)\right)=\gamma_{2}(\tau), \\
& \gamma_{3}=\sqrt{1-\gamma_{1}^{2}(\tau)-\gamma_{2}^{2}(\tau)}, \\
& t=t_{0}+I_{1} I_{2} \int \frac{d \tau}{\sqrt{1-\gamma_{1}^{2}(\tau)-\gamma_{2}^{2}(\tau)}} .
\end{aligned}
$$

If

$$
\begin{aligned}
& \sqrt{a_{1}-b_{1}} I_{1}=\sqrt{a_{2}-b_{2}} I_{2}=\alpha, \quad \tau_{1}=\tau_{2}=0, \\
& \frac{h_{1}+h_{2}+a_{1}+a_{2}+a_{3}+a_{4}}{I_{1}-I_{2}}=k^{2}>0,
\end{aligned}
$$

then

$$
t=t_{0}+I_{1} I_{2} \int \frac{d \tau}{\sqrt{1-k^{2} \sin ^{2}(\alpha \tau)}} .
$$

In the most general case the analytical character of the solutions is essentially more complex. Hence the proof of statement (c) follows. This completes the proof of Theorem 1.

Remark 5(a) In all the known integrable cases studied before our work condition (16) holds everywhere, and consequently we have local first integrals everywhere, but the known integrals are globally defined.

(b) In the previous results we determine $\gamma$ as a function of time through the quadratures. In general these quadratures contain elliptic integrals, consequently to deduce the explicit form of the time dependence require previously to invert these integral. To obtain the time dependence of the angular velocity vector we use the constraint $\omega_{3}=0$, and the general integrals $I_{1} \omega_{1}-\mu_{2}=0$ and $I_{2} \omega_{2}+\mu_{1}=0$.

\section{Proof of Theorem 2 and Corollary 1}

We start with some preliminary computations that are necessary for proving Theorem 2 and Corollary 3 (see [18]).

First, in view of (23), using the constraint $\gamma_{1} \omega_{1}+\gamma_{2} \omega_{2}+\gamma_{3} \omega_{3}=\dot{y}+\dot{x} \cos z=$ 0 and $\gamma_{1}^{2}+\gamma_{2}^{2}+\gamma_{3}^{2}=1$ we deduced the relations

$$
\omega_{1}=\frac{p \Psi_{2} \gamma_{2}-I_{2} \Psi_{1} \gamma_{1} \gamma_{3}}{p\left(I_{1} \gamma_{2}^{2}+I_{2} \gamma_{1}^{2}\right)}, \quad \omega_{2}=-\frac{p \Psi_{2} \gamma_{1}+I_{1} \Psi_{1} \gamma_{2} \gamma_{3}}{p\left(I_{1} \gamma_{2}^{2}+I_{2} \gamma_{1}^{2}\right)}, \quad \omega_{3}=\frac{\Psi_{1}}{p} .
$$


Consequently

$$
\langle I \omega, \omega\rangle=\frac{\Psi_{1}^{2}+\Psi_{2}^{2}}{I_{1} \gamma_{2}^{2}+I_{2} \gamma_{1}^{2}}
$$

From (45) the Poisson equations become (25), which in view of (4) we get

$$
\dot{x}=\frac{\Psi_{1}}{\sin ^{2} z p}, \quad \dot{y}=-\frac{\cos z \Psi_{1}}{\sin ^{2} z p}, \quad \dot{z}=\frac{\Psi_{2} p+\left(I_{1}-I_{2}\right) \Psi_{1} \cos x \sin x \cos z}{p \sin z\left(I_{1} \cos ^{2} x+I_{2} \sin ^{2} x\right)} .
$$

From the Poisson equations (25) and equation (47) we obtain the relation

$$
\begin{aligned}
& \frac{d G(\gamma)}{d t}=\left(\gamma_{2} \omega_{3}-\gamma_{3} \omega_{2}\right) \frac{\partial G}{\partial \gamma_{1}}+\left(\gamma_{3} \omega_{1}-\gamma_{1} \omega_{3}\right) \frac{\partial G}{\partial \gamma_{2}}+\left(\gamma_{1} \omega_{2}-\gamma_{2} \omega_{1}\right) \frac{\partial G}{\partial \gamma_{3}} \\
& =\frac{\left(\left(I_{2}-I_{1}\right)\left\langle\gamma, \frac{\partial G}{\partial \gamma}\right\rangle \gamma_{1} \gamma_{2}+I_{1} \gamma_{2} \frac{\partial G}{\partial \gamma_{1}}-I_{2} \gamma_{1} \frac{\partial G}{\partial \gamma_{2}}\right) \Psi_{1}+p \Psi_{2}\left(\gamma_{3}\left\langle\gamma, \frac{\partial G}{\partial \gamma}\right\rangle-\frac{\partial G}{\partial \gamma_{3}}\right)}{p\left(I_{1} \gamma_{2}^{2}+I_{2} \gamma_{1}^{2}\right)} \\
& =\frac{\Psi_{1}}{p\left(I_{1} \gamma_{2}^{2}+I_{2} \gamma_{1}^{2}\right)}\left(\left(I_{1} \cos ^{2} x+I_{2} \sin ^{2} x\right) \frac{\partial G}{\partial x}+\left(I_{1}-I_{2}\right) \cos x \sin x \cos z \sin z \frac{\partial G}{\partial z}\right) \\
& +\frac{p \Psi_{2} \sin z}{p\left(I_{1} \gamma_{2}^{2}+I_{2} \gamma_{1}^{2}\right)} \frac{\partial G}{\partial z} .
\end{aligned}
$$

Thus, using that $\Psi_{1}$ and $\Psi_{2}$ are arbitrary functions we get that

$$
\begin{aligned}
& \left(I_{2}-I_{1}\right)\left\langle\gamma, \frac{\partial G}{\partial \gamma}\right\rangle \gamma_{1} \gamma_{2}+I_{1} \gamma_{2} \frac{\partial G}{\partial \gamma_{1}}-I_{2} \gamma_{1} \frac{\partial G}{\partial \gamma_{2}} \\
& =\left(I_{1} \cos ^{2} x+I_{2} \sin ^{2} x\right) \frac{\partial G}{\partial x}+\left(I_{1}-I_{2}\right) \cos x \sin x \cos z \sin z \frac{\partial G}{\partial z} \\
& \gamma_{3}\left\langle\gamma, \frac{\partial G}{\partial \gamma}\right\rangle-\frac{\partial G}{\partial \gamma_{3}}=\sin z \frac{\partial G}{\partial z} .
\end{aligned}
$$


Now we calculate the derivative of $\Psi_{1}=\Psi_{j}(x, z)$ for $j=1,2$ along the solutions of (47) and using (21) we obtain

$$
\begin{aligned}
& \frac{d \Psi_{2}}{d t}=\frac{\Psi_{1}}{p\left(I_{1} \gamma_{2}^{2}+I_{2} \gamma_{1}^{2}\right)}\left(I_{1} \cos ^{2} x+I_{2} \sin ^{2} x\right) \frac{\partial \Psi_{2}}{\partial x} \\
& +\frac{1}{\left(I_{1} \gamma_{2}^{2}+I_{2} \gamma_{1}^{2}\right)}\left(I_{1}-I_{2}\right) \cos x \sin x \cos z \sin z \frac{\partial \Psi_{2}}{\partial z} \\
& +\frac{\sin z}{2\left(I_{1} \gamma_{2}^{2}+I_{2} \gamma_{1}^{2}\right)} \frac{\partial \Psi_{2}^{2}}{\partial z} \\
& =\Theta+\frac{\sin z}{\left(I_{1} \gamma_{2}^{2}+I_{2} \gamma_{1}^{2}\right)} \frac{\partial \Psi}{\partial z} \\
& =\Theta+\sin z \frac{\partial}{\partial z}\left(\frac{\Psi}{I_{1} \gamma_{2}^{2}+I_{2} \gamma_{1}^{2}}\right)+2 \cos z \frac{\Psi}{I_{1} \gamma_{2}^{2}+I_{2} \gamma_{1}^{2}}, \\
& \frac{d}{d t} \Psi_{1}=\frac{\Psi_{1}}{p\left(I_{1} \gamma_{2}^{2}+I_{2} \gamma_{1}^{2}\right)}\left(\left(I_{1} \cos ^{2} x+I_{2} \sin ^{2} x\right) \frac{\partial \Psi_{1}}{\partial x}\right. \\
& \left.+\left(I_{1}-I_{2}\right) \cos x \sin x \cos z \sin z \frac{\partial \Psi_{1}}{\partial z}\right) \\
& +\frac{p \Psi_{2} \sin z}{p\left(I_{1} \gamma_{2}^{2}+I_{2} \gamma_{1}^{2}\right)} \frac{\partial \Psi_{1}}{\partial z} \\
& =\Theta+\frac{1}{p\left(I_{1} \gamma_{2}^{2}+I_{2} \gamma_{1}^{2}\right)}\left(I_{1} \cos ^{2} x+I_{2} \sin ^{2} x\right) \frac{\partial \Psi}{\partial x} \\
& +\frac{1}{p\left(I_{1} \gamma_{2}^{2}+I_{2} \gamma_{1}^{2}\right)}\left(I_{1}-I_{2}\right) \cos x \sin x \cos z \sin z \frac{\partial \Psi}{\partial z} \\
& =\Theta+\frac{1}{\left(I_{1} \gamma_{2}^{2}+I_{2} \gamma_{1}^{2}\right)}\left(\left(I_{2}-I_{1}\right)\left\langle\gamma, \frac{\partial \Psi}{\partial \gamma}\right\rangle \gamma_{1} \gamma_{2}+I_{1} \gamma_{2} \frac{\partial \Psi}{\partial \gamma_{1}}-I_{2} \gamma_{1} \frac{\partial \Psi}{\partial \gamma_{2}}\right) \\
& =\Theta+\left(I_{2}-I_{1}\right) \gamma_{1} \gamma_{2}\left(\left\langle\gamma, \frac{\partial}{\partial \gamma}\left(\frac{\Psi}{I_{1} \gamma_{2}^{2}+I_{2} \gamma_{1}^{2}}\right)\right\rangle+2 \frac{\Psi}{I_{1} \gamma_{2}^{2}+I_{2} \gamma_{1}^{2}}\right)+ \\
& I_{1} \gamma_{2} \frac{\partial}{\partial \gamma_{1}}\left(\frac{\Psi}{I_{1} \gamma_{2}^{2}+I_{2} \gamma_{1}^{2}}\right)-I_{2} \gamma_{1} \frac{\partial}{\partial \gamma_{2}}\left(\frac{\Psi}{I_{1} \gamma_{2}^{2}+I_{2} \gamma_{1}^{2}}\right) \text {, }
\end{aligned}
$$

where $\Psi=\frac{1}{2}\left(\Psi_{1}^{2}+\Psi_{3}^{2}\right)$.

Proof (Proof of Theorem 2) After some tedious computations from (11), (45), (46), (47), (48) and (4) we deduce the relations

$$
\begin{aligned}
\frac{d F_{1}}{d t} & =\frac{d}{d t}\left(I_{1} \omega_{1} \gamma_{2}-I_{2} \omega_{2} \gamma_{1}\right)-\frac{d}{d t} \Psi_{2} \\
& =-\Theta-\sin z \frac{\partial}{\partial z}\left(U+\frac{\Psi}{I_{1} \gamma_{2}^{2}+I_{2} \gamma_{1}^{2}}\right)+\cos z\left(\langle I \omega, \omega\rangle-\frac{2 \Psi}{I_{1} \gamma_{2}^{2}+I_{2} \gamma_{1}^{2}}\right)
\end{aligned}
$$


Thus $\left.\frac{d F_{1}}{d t}\right|_{F_{1}=F_{2}=0}=-\Theta$. Moreover,

$$
\begin{aligned}
\frac{d F_{2}}{d t}= & \frac{d(p \omega)}{d t}-\frac{d \Psi_{1}}{d t} \\
= & -\Theta-\left(I_{2}-I_{1}\right) \gamma_{1} \gamma_{2}\left(\left\langle\gamma, \frac{\partial}{\partial \gamma}\left(U+\frac{\Psi}{I_{1} \gamma_{2}^{2}+I_{2} \gamma_{1}^{2}}\right)\right\rangle+\langle I \omega, \omega\rangle-\frac{2 \Psi}{I_{1} \gamma_{2}^{2}+I_{2} \gamma_{1}^{2}}\right) \\
& +I_{1} \gamma_{2} \frac{\partial}{\partial \gamma_{1}}\left(U+\frac{\Psi}{I_{1} \gamma_{2}^{2}+I_{2} \gamma_{1}^{2}}\right)-I_{2} \gamma_{1} \frac{\partial}{\partial \gamma_{2}}\left(U+\frac{\Psi}{I_{1} \gamma_{2}^{2}+I_{2} \gamma_{1}^{2}}\right) .
\end{aligned}
$$

Hence $\left.\frac{d F_{2}}{d t}\right|_{F_{1}=F_{2}=0}=-\Theta$.

Here we apply the relation

$$
\langle I \omega, \omega\rangle=2(h-U)=\frac{\Psi_{1}^{2}+\Psi_{2}^{2}}{I_{1} \gamma_{2}^{2}+I_{2} \gamma_{1}^{2}} \quad \text { with } \quad h=\text { constant }
$$

obtained from energy integral in view of (46), and

$$
\begin{aligned}
& \frac{d(p \omega)}{d t}=\left(I_{1}-I_{2}\right) \gamma_{1} \gamma_{2}\left(\left\langle\gamma, \frac{\partial U}{\partial \gamma}\right\rangle+\langle I \omega, \omega\rangle\right)+I_{1} \gamma_{2} \frac{\partial U}{\partial \gamma_{1}}-I_{2} \gamma_{1} \frac{\partial U}{\partial \gamma_{2}} \\
& \frac{d}{d t}\left(I_{1} \omega_{1} \gamma_{2}-I_{2} \omega_{2} \gamma_{1}\right)=-\gamma_{3}\left\langle\gamma, \frac{\partial U}{\partial \gamma}\right\rangle+\frac{\partial U}{\partial \gamma_{3}}+\gamma_{3}\langle I \omega, \omega\rangle
\end{aligned}
$$

which we deduce from (12) and (13). Consequently in view of (21) we have $\left.\frac{d F_{j}}{d t}\right|_{F_{1}=F_{2}=0}=0$, for $j=1,2$. Hence $F_{1}=0$ and $F_{2}=0$ are general integrals. This proves statement (a) of the theorem.

To prove statement (b), first we observe that if $\Psi_{1}^{2}+\Psi_{2}^{2}=2 \Psi(x)$, then from (4) we obtain that $\frac{d \Psi_{2}}{d t}=\Theta$. Thus if (21) holds then $\Psi_{2}=K_{4}=$ arbitrary constant. Consequently, from statement (a), we obtain the first integral $I_{1} \omega_{1} \gamma_{2}-I_{2} \omega_{2} \gamma_{1}=K_{4}$. By considering the energy integral, and in view of the Euler-Jacobi Theorem we obtain the proof of statement (b).

Now we prove statement (c). If $I_{1} \neq I_{2}$ and $\Psi_{1}^{2}+\Psi_{2}^{2}=2 \Psi\left(\frac{I_{1} \gamma_{2}^{2}+I_{2} \gamma_{1}^{2}}{\gamma_{3}^{2}}\right)$, then from (4) it follows the proof of this statement by considering that the function $\Psi\left(\frac{I_{1} \gamma_{2}^{2}+I_{2} \gamma_{1}^{2}}{\gamma_{3}^{2}}\right)$ is a solution of the equation

$$
\begin{aligned}
0 & =\left(I_{2}-I_{1}\right)\left\langle\gamma, \frac{\partial \Psi}{\partial \gamma}\right\rangle \gamma_{1} \gamma_{2}+I_{1} \gamma_{2} \frac{\partial \Psi}{\partial \gamma_{1}}-I_{2} \gamma_{1} \frac{\partial \Psi}{\partial \gamma_{2}} \\
& =\left(I_{1} \cos ^{2} x+I_{2} \sin ^{2} x\right) \frac{\partial \Psi}{\partial x}+\left(I_{1}-I_{2}\right) \cos x \sin x \cos z \sin z \frac{\partial \Psi}{\partial z}
\end{aligned}
$$


For proving statement (d), first we observe that the partial differential equation (21) for $I_{1}=I_{2}$ takes the form

$$
\frac{\partial \Psi_{2}}{\partial x}-\sin z \sqrt{\alpha+(1-\alpha) \cos ^{2} z} \frac{\partial \Psi_{1}}{\partial z}=0, \quad \alpha=\frac{I_{3}}{I_{1}} .
$$

Hence

$$
\frac{\partial}{\partial x}\left(\frac{\Psi_{2}}{\sin z \sqrt{\alpha+(1-\alpha) \cos ^{2} z}}\right)-\frac{\partial \Psi_{1}}{\partial z}=0 .
$$

Thus we get that

$$
\Psi_{2}=\sin z \sqrt{\alpha+(1-\alpha) \cos ^{2} z} \frac{\partial S}{\partial z}, \quad \Psi_{1}=\frac{\partial S}{\partial x} .
$$

where $S=S(x, z)$ is an arbitrary function. If $\Psi_{1}^{2}+\Psi_{2}^{2}=2 \Psi\left(z, K_{3}, K_{4}\right)$, then from (21) we deduce that $\frac{d \Psi_{1}}{d t}=\Theta$, consequently in view of (21) we obtain that $\Psi_{1}=K_{4}=$ arbitrary constant.

In particular if $S=S_{1}(z)+K_{4} x$, then $\Psi_{1}=K_{4}$, and consequently from (51) we obtain the first integral $\sqrt{\alpha+(1-\alpha) \cos ^{2} z} \omega_{3}=K_{4}$. By considering the energy integral, and in view of the Euler-Jacobi Theorem we obtain the proof of statement (c).

Finally we observe that differential system (47) when $\Psi_{1}=K_{4}$ and $I_{1}=I_{2}$ takes the form

$$
\begin{aligned}
& \dot{x}=\frac{K_{4}}{\sin ^{2} z \sqrt{\alpha+(1-\alpha) \cos ^{2} z}}, \\
& \dot{y}=\frac{-\cos z K_{4}}{\sin ^{2} z \sqrt{\alpha+(1-\alpha) \cos ^{2} z}}, \\
& \dot{z}=\frac{\sqrt{\alpha+(1-\alpha) \cos ^{2} z}}{I_{1}} S_{1}^{\prime} .
\end{aligned}
$$

The solutions of these equations are easy to obtain because it is of separable variables. This completes the proof of Theorem 2 .

Proof (Proof of Corollary 3) The proof follows from the relations (48).

\section{Proof of Theorem 1 and Corollary 2}

Proof (Proof of Theorem 1) From (12) and after an easy computation we have

$$
\frac{d}{d t}\left(\|\gamma \wedge I \omega\|^{2}\right)=-2\left\langle\frac{\partial U}{\partial \gamma}, \gamma \wedge I \omega\right\rangle .
$$

The function $\frac{1}{2}\|\gamma \wedge I \omega\|^{2}-W\left(\gamma_{1}, \gamma_{2}, \gamma_{3}\right)$ is a first integral if and only if

$$
\left\langle\frac{\partial U}{\partial \gamma}, \gamma \wedge I \omega\right\rangle=\left\langle\frac{\partial W}{\partial \gamma}, \gamma \wedge \omega\right\rangle
$$


consequently

$$
\left\langle I\left(\frac{\partial U}{\partial \gamma} \wedge \gamma\right)-\frac{\partial W}{\partial \gamma} \wedge \gamma, \omega\right\rangle=0
$$

Thus, by considering that $\langle\gamma, \omega\rangle=0$, we have

$$
I\left(\frac{\partial U}{\partial \gamma} \wedge \gamma\right)-\frac{\partial W}{\partial \gamma} \wedge \gamma=\lambda \gamma
$$

or equivalently

$$
\frac{\partial U}{\partial \gamma} \wedge \gamma-I^{-1}\left(\frac{\partial W}{\partial \gamma} \wedge \gamma\right)=\lambda I^{-1} \gamma
$$

where $\lambda=\lambda\left(\gamma_{1}, \gamma_{2}, \gamma_{3}\right)$ is an arbitrary function.

To solve the partial differential equations (52) when $\Delta \neq 0$ we use the relations

$$
\frac{\partial f}{\partial \gamma_{j}}=2 \gamma_{j}\left(\frac{\partial \bar{f}}{\partial \tau_{1}}+I_{j} \frac{\partial \bar{f}}{\partial \tau_{2}}+\frac{1}{I_{j}} \frac{\partial \bar{f}}{\partial \tau_{3}}\right)
$$

for $j=1,2,3$, where $f=f\left(\gamma_{1}, \gamma_{2}, \gamma_{3}\right)=\bar{f}\left(\tau_{1}, \tau_{2}, \tau_{3}\right)$. Hence

$$
\begin{aligned}
& \frac{\partial f}{\partial \gamma} \wedge \gamma= \\
& v\left(\frac{\left(I_{2}-I_{3}\right)}{\gamma_{1}}\left(\frac{\partial \bar{f}}{\partial \tau_{2}}-\frac{I_{1}}{|I|} \frac{\partial \bar{f}}{\partial \tau_{3}}\right), \frac{\left(I_{3}-I_{1}\right)}{\gamma_{2}}\left(\frac{\partial \bar{f}}{\partial \tau_{2}}-\frac{I_{2}}{|I|} \frac{\partial f}{\partial \tau_{3}}\right), \frac{\left(I_{1}-I_{2}\right)}{\gamma_{3}}\left(\frac{\partial \bar{f}}{\partial \tau_{2}}-\frac{I_{3}}{|I|} \frac{\partial \bar{f}}{\partial \tau_{3}}\right)\right)
\end{aligned}
$$

where $v=2 \gamma_{1} \gamma_{2} \gamma_{3}$. After some computations we obtain

$$
\begin{gathered}
I\left(\frac{\partial U}{\partial \gamma} \wedge \gamma\right)-\frac{\partial W}{\partial \gamma} \wedge \gamma=v\left(\frac{\left(I_{2}-I_{3}\right)}{\gamma_{1}} \Phi_{1}, \frac{\left(I_{3}-I_{1}\right)}{\gamma_{2}} \Phi_{2}, \frac{\left(I_{1}-I_{2}\right)}{\gamma_{3}} \Phi_{3}\right)=\lambda \gamma \\
\Phi_{j}=I_{j} \frac{\partial U}{\partial \tau_{2}}-\frac{I_{j}^{2}}{|I|} \frac{\partial U}{\partial \tau_{3}}-\frac{\partial W}{\partial \tau_{2}}+\frac{I_{j}}{|I|} \frac{\partial W}{\partial \tau_{3}}
\end{gathered}
$$

for $j=1,2,3$. Thus

$$
\left(I_{2}-I_{3}\right) \Phi_{1}=\frac{\lambda}{2 \gamma_{1} \gamma_{2} \gamma_{3}} \gamma_{1}^{2}, \quad\left(I_{3}-I_{1}\right) \Phi_{2}=\frac{\lambda}{2 \gamma_{1} \gamma_{2} \gamma_{3}} \gamma_{2}^{2}, \quad\left(I_{1}-I_{2}\right) \Phi_{3}=\frac{\lambda}{2 \gamma_{1} \gamma_{2} \gamma_{3}} \gamma_{3}^{2} .
$$

Hence, by using the relations

$$
\begin{aligned}
\frac{I_{2}-I_{3}}{I_{1}}+\frac{I_{3}-I_{1}}{I_{2}}+\frac{I_{1}-I_{2}}{I_{3}} & =\frac{I_{1}^{2}\left(I_{3}-I_{2}\right)+I_{2}^{2}\left(I_{1}-I_{3}\right)+I_{3}^{2}\left(I_{2}-I_{1}\right)}{I_{1} I_{2} I_{3}} \\
& =\frac{\left(I_{1}-I_{2}\right)\left(I_{2}-I_{3}\right)\left(I_{3}-I_{1}\right)}{I_{1} I_{2} I_{3}} \neq 0, \\
I_{1}^{3}\left(I_{2}-I_{3}\right)+I_{2}^{3}\left(I_{3}-I_{1}\right)+I_{3}^{3}\left(I_{1}-I_{2}\right) & =-\left(I_{1}-I_{2}\right)\left(I_{2}-I_{3}\right)\left(I_{3}-I_{1}\right)\left(I_{1}+I_{2}+I_{3}\right),
\end{aligned}
$$


we deduce that

$$
\begin{aligned}
& \frac{\left(I_{1}-I_{2}\right)\left(I_{2}-I_{3}\right)\left(I_{3}-I_{1}\right)}{I_{1} I_{2} I_{3}} \frac{\partial U}{\partial \tau_{3}}=\frac{\lambda}{\gamma_{1} \gamma_{2} \gamma_{3}} \tau_{1}, \\
& \frac{\left(I_{1}-I_{2}\right)\left(I_{2}-I_{3}\right)\left(I_{3}-I_{1}\right)}{I_{1} I_{2} I_{3}} \frac{\partial W}{\partial \tau_{2}}=\frac{\lambda}{\gamma_{1} \gamma_{2} \gamma_{3}} \tau_{3}, \\
& \frac{\left(I_{1}-I_{2}\right)\left(I_{2}-I_{3}\right)\left(I_{3}-I_{1}\right)}{I_{1} I_{2} I_{3}}\left(I_{1} I_{2} I_{3} \frac{\partial U}{\partial \tau_{2}}+\frac{\partial W}{\partial \tau_{3}}-\left(I_{1}+I_{2}+I_{3}\right) \frac{\partial U}{\partial \tau_{3}}\right)= \\
& -\frac{\lambda \tau_{2}}{\gamma_{1} \gamma_{2} \gamma_{3}} .
\end{aligned}
$$

Denoting by $\tilde{\nu}=\frac{I_{1} I_{2} I_{3} \lambda}{\left(I_{1}-I_{2}\right)\left(I_{2}-I_{3}\right)\left(I_{3}-I_{1}\right) \gamma_{1} \gamma_{2} \gamma_{3}}$ from (53) we easily obtain (30). This is the proof of statement (a).

Now we consider the case $\Delta \neq 0$ and $\mu=0$. The equations of motion for a rigid body without constraints are the Euler-Poisson equations

$$
I \dot{\omega}=I \omega \wedge \omega+\gamma \wedge \frac{\partial U}{\partial \gamma}, \quad \dot{\gamma}=\gamma \wedge \omega
$$

The necessary and sufficient conditions for the existence of a first integral $K_{4}$ are obtained from (52) with $\lambda=0$, because the system is free of constraints. Hence, from (53) we get the condition (31). This completes the proof of the theorem.

Proof (Proof of Corollary 2) From (30) and considering that $\tau_{1}=1$ we have

$$
\begin{aligned}
& \frac{\partial W}{\partial \tau_{2}}=\tau_{3} \frac{\partial U}{\partial \tau_{3}} \\
& \frac{\partial W}{\partial \tau_{3}}=-|I| \frac{\partial U}{\partial \tau_{2}}+\left(-\tau_{2}+\left(I_{1}+I_{2}+I_{3}\right)\right) \frac{\partial U}{\partial \tau_{3}}
\end{aligned}
$$

The compatibility condition of this system is given by equation (33). Hence if $U$ satisfies the equation (33), then the function $W$ is obtained integrating (54). Consequently from (29) the require first integral takes the form (32). In short, statement (a) is proved.

Under the assumptions of statement (b) the potential function $U$ must satisfy the equations (31). So $\tilde{\nu}=0$. Now the proof of statement (b) is easy to obtain from the proof of the previous statement. Thus the theorem is proved.

Proof (Proof of Corollary 3) The potential function

$$
U=a_{0}+a_{1} \tau_{2}+a_{2}\left(\tau_{2}^{2}-|I| \tau_{3}\right)+\frac{a_{4}}{a_{7}+a_{5} \tau_{2}+a_{6} \tau_{3}},
$$

where

$$
a_{6}=\frac{a_{4}\left(|I| a_{4}-a_{5}\left(I_{1}+I_{2}+I_{3}\right)\right.}{a_{5}},
$$


for arbitrary $a_{0}, a_{1}, a_{2}, a_{3}, a_{4}, a_{5} \neq 0$, is a particular solution of (33). Then the first integral (32) in this case becomes

$\Phi_{4}=\frac{1}{2}|| \gamma \wedge I \omega \|^{2}-|I| \alpha\left(\tau_{2} \tau_{3}+\left(I_{1}+I_{2}+I_{3}\right) \tau_{3}\right)+|I| \beta \tau_{3}+\frac{a_{3}\left(a_{4} \tau_{2}+a_{6}\right)}{a_{6}+a_{4} \tau_{2}+a_{5} \tau_{3}}$.

Consequently, from (54) we get that

$$
W=\frac{a_{4}\left(a_{5} \tau_{2}+a_{7}\right)}{a_{7}+a_{5} \tau_{2}+a_{6} \tau_{3}}+|I| a_{2}\left(\tau_{2} \tau_{3}+\left(I_{1}+I_{2}+I_{3} \tau_{3}\right)\right)-|I| a_{1} \tau_{3} .
$$

We denote by $w=a_{7}+a_{5} \tau_{2}+a_{6} \tau_{3}$. We study the following particular cases

$$
\begin{array}{llll}
a_{4}=\frac{\alpha_{3}}{r_{1}}, & a_{5}=r_{1}, & a_{6}=r_{1} I_{1} I_{2}, & a_{7}=-r_{1}\left(I_{3}+I_{2}\right) \Longrightarrow w=\gamma_{1}^{2}, \\
a_{4}=\frac{\alpha_{4}}{r_{2}}, & a_{5}=r_{2}, & a_{6}=r_{2} I_{1} I_{3}, & a_{7}=-r_{2}\left(I_{3}+I_{1}\right) \Longrightarrow w=\gamma_{2}^{2}, \\
a_{4}=\frac{\alpha_{5}}{r_{3}}, & a_{5}=r_{3}, & a_{6}=r_{3} I_{1} I_{3}, & a_{7}=-r_{3}\left(I_{3}+I_{1}\right) \Longrightarrow w=\gamma_{3}^{2},
\end{array}
$$

where $r_{1}, r_{2}, r_{3}$ are constants given in (28). By considering that (54) and (33) are linear partial differential equations we obtain that the linear combination of the functions

$$
\begin{aligned}
U_{1} & =\frac{1}{\gamma_{1}^{2}}, \quad U_{2}=\frac{1}{\gamma_{2}^{2}}, \quad U_{3}=\frac{1}{\gamma_{3}^{2}}, \\
W_{1} & =\frac{\left.I_{2}\left(1-\gamma_{2}^{2}\right)+I_{3}\left(1-\gamma_{3}^{2}\right)+I_{1} \gamma_{1}^{2}\right)}{\gamma_{1}^{2}}, \\
W_{2} & =\frac{\left.I_{1}\left(1-\gamma_{1}^{2}\right)+I_{3}\left(1-\gamma_{3}^{2}\right)+I_{2} \gamma_{2}^{2}\right)}{\gamma_{2}^{2}}, \\
W_{3} & =\frac{\left.I_{2}\left(1-\gamma_{2}^{2}\right)+I_{1}\left(1-\gamma_{1}^{2}\right)+I_{3} \gamma_{3}^{2}\right)}{\gamma_{3}^{2}},
\end{aligned}
$$

are solutions of these equations. Therefore for the potential function (35) or what is the same

$$
\begin{aligned}
U & =a_{0}+a_{1} \tau_{2}+a_{2}\left(\tau_{2}^{2}-|I| \tau_{3}\right)+\alpha_{3} U_{1}+\alpha_{4} U_{2}+\alpha_{5} U_{3} \\
W & =|I| a_{2}\left(\tau_{2} \tau_{3}+\left(I_{1}+I_{2}+I_{3} \tau_{3}\right)\right)+|I| a_{1} \tau_{3}+\alpha_{4} W_{1}+\alpha_{5} W_{2}+\alpha_{6} W_{3}
\end{aligned}
$$

we obtain the first integral

$$
\begin{aligned}
\Phi_{4}= & \frac{1}{2}|| \gamma \wedge I \omega \|^{2}+-|I| a_{2}\left(\tau_{2} \tau_{3}+\left(I_{1}+I_{2}+I_{3} \tau_{3}\right)\right)+|I| a_{1} \tau_{3}+ \\
& \frac{\alpha_{3}\left(I_{2}\left(1-\gamma_{2}^{2}\right)+I_{3}\left(1-\gamma_{3}^{2}\right)+I_{1} \gamma_{1}^{2}\right)}{\gamma_{1}^{2}}+\frac{\alpha_{4}\left(\left(I_{1}\left(1-\gamma_{1}^{2}\right)+I_{3}\left(1-\gamma_{3}^{2}\right)+I_{2} \gamma_{2}^{2}\right)\right.}{\gamma_{2}^{2}} \\
& +\frac{\alpha_{5}\left(\left(I_{2}\left(1-\gamma_{2}^{2}\right)+I_{1}\left(1-\gamma_{1}^{2}\right)+I_{3} \gamma_{3}^{2}\right)\right)}{\gamma_{3}^{2}}
\end{aligned}
$$

where $\alpha_{j}$ for $j=3,4,5$ are constants. 


\section{Acknowledgements}

The first author is partially supported by a MICINN/FEDER grant number MTM2009-03437, an AGAUR grant number 2009SGR-410, ICREA Academia and two FPZ-PEOPLE-2012-IRSES numbers 316338 and 318999. The second author was partly supported by the Spanish Ministry of Education through projects TSI2007-65406-C03-01 "E-AEGIS" and Consolider CSD2007-00004 "ARES".

\section{References}

1. V.I. Arnold, Dynamical systems III, Springer-Verlag, 1996.

2. A.M. Bloch, Nonholonomic Mechanics and Control, Springer-Verlag, 2003.

3. A.V. Borisov, A.A. Killing and I.S. Mamaev, Multiparticle Systems. The algebra of integrals and integrable cases, Regular and chaotic dynamics 14 (2009), 18-41.

4. A.V. Borisov and I.S. Mamaev, Dynamics of a rigid body, Institut Kompyuternykh Issledobanyi, 2005 (in Russian).

5. A.V. Borisov and I.S. Mamaev, Conservation laws, hierarchy of dynamics and explicit integration of nonholonomic systems, Regular and chaotic dynamics 13 (2008), 443490.

6. V. Dragović , B. Gajić and B. Jovanović, Generalizations of classical integrable nonholonomic rigid body systems, J. Phys. A: Math. Gen. 31 (1998), 9861-9869.

7. Y. N. Fedorov, A. Maciejewski and M. Przybylska The Posisson equations in the nonholonomic Suslov problem: integrability, meromorphic and hypergeometric solutions, J. Nonlinear Sci. 22 (2009) 2231-2259.

8. Y. N. Fedorov, Two integrable nonholonomic systems in Classical Dynamics, Vestnik Moskow. Univ. Ser. I Mat. Mekh. 4 (1989), 38-41.

9. Y.N. Fedorov and B. Jovanovic, Nonholonomic LR systems as generalized Chaplygin systems with an invariant measure and flows on homogeneous space, J. Nonlinear Sci. 14,(2004) 341-381.

10. A. Goriely, Integrability and nonintegrability of dynamical systems, Advances Series in Nonlinear Dynamics 19, World Scientific Publishing Co., Inc., River Edge, NJ, 2001.

11. E.I. Kharlamova-Zabelina, Rapid rotation of a rigid body about a fixed point under the presence of a nonholonomic constraints, (Russian) Vestnik Moskovsk. Univ., Ser. Math. Mekh., Astron., Fiz.Khim. 6 (1957), 25-34.

12. V.V. Kozlov, On the integration theory of the equations of nonholonomic mechanics, Regular and chaotic dynamics 7 (2002), 161-172.

13. V.V. Kozlov, Symmetries, Topology and Resonances in Hamiltonian Mechanics, 1995 Springer-Verlag, Berlin,

14. V.V. Kozlov, Dynamical systems X, General theory of vortices, Spriger, 2003.

15. V.V. Kozlov, Several problems on dynamical systems and mechanics, Nonlinearity 21 (2008), 149-155.

16. A.J. Maciejewski and M. Przybylska, Non-integrability of the Suslov problem, Regular and Chaotic Dynamics 7 (2002), 73-80.

17. G.G. Okuneva, A qualitative investigation of an integrable variant of the nonholonomic Suslov problem, (Russian) Vestnik Moskov. Univ. Ser. I Mat. Mekh. (1987), 59-64.

18. R. Ramirez and N. Sadovskaia, Cartesian approach for constrained mechanical systems, Arxiv 2010.

19. G.K Suslov, Theoretical mechanics. M.-L.: Gostechizdat, 1946.

20. L.E. Veselova, Novie sluchai integriruemosti uravneniy dvijenia tverdogo tela pri nalichii negolonomnoi sviazi. Sbornik Geometria, dif. uravnenia i mekhanika MGU (1986), 6468.

21. A.P. Veselov, L.E. Veselova Flows on Lie group with nonholonomic constraint and integrable non-Hamiltonian systems. Funct. Anal. Appl. 20 (1986), 308-309. 